\title{
Actinistian gular plates from the Cretaceous of Mexico and the problems assigning gular plates taxonomically
}

\author{
Hans-Peter Schultze ${ }^{1}$ and Katia Adriana González-Rodríguez ${ }^{2}$ \\ ${ }^{1}$ University of Kansas, Natural History Museum and Biodiversity Institute, Lawrence, Kansas, USA \\ ${ }^{2}$ Instituto de Ciencias Básicas e Ingeniería, Museo de Paleontología, Centro de Investigaciones Biológicas, \\ Universidad Autónoma del Estado de Hidalgo, Mineral de la Reforma, Hidalgo, Mexico \\ Correspondence to: Hans-Peter Schultze (hp1937@ku.edu)
}

Received: 7 January 2016 - Revised: 22 February 2016 - Accepted: 3 March 2016 - Published: 15 April 2016

\begin{abstract}
Two gular plates of an indeterminate actinistian are described from the Cretaceous of Muhi quarry, Hidalgo state, in central Mexico. Their narrow shape belongs to the few actinistian gular plates with a high length/width ratio (above 4) in contrast to most known actinistian gular plates with a length / width ratio below 4 . The gulars of Muhi quarry are assigned, with caution, to the actinistian family Mawsoniidae on the basis of the length / width ratio (around 4.5) of the gular, which can be found in three other genera of the family. There are not enough characteristics in actinistian gular plates alone to erect a new genus or species. Noteworthy is the large size of the Muhi gulars that corresponds to a body length of about $1.6 \mathrm{~m}$ - the length of Latimeria today. The gulars are preserved in association with a basibranchial tooth plate and a few neural spines. This is the third actinistian record from the Cretaceous of Mexico.
\end{abstract}

\section{Introduction}

There are many Cretaceous marine localities in Mexico with fossil fishes (González-Rodríguez et al., 2013a). Different chondrichthyans and a rich actinopterygian fauna are known, but only two actinistian are recorded: one from Tlayúa (Espinosa-Arrubarrena et al., 1996 [specimen lost]) and another from Vallecillo (Schultze et al., 2010). The Muhi quarry of Hidalgo state in central Mexico is Albian-Cenomanian in age, more likely Albian based on the ammonite record (see González-Rodríguez et al., 2013b, p. 459). Its rich teleostean fauna and few teleosteomorph aspidorhynchid and chondrichthyan remains are considered as unique (GonzálezRodríguez et al., 2013a). Holosteans have not been discov- ered yet. The composition of the fish fauna clearly distinguishes the Muhi locality from other Mexican Cretaceous localities, specifically from the slightly older Tlayúa quarry in Puebla state with many holosteans (Amiiformes, Semionotiformes, and Macrosemiiformes) and Pycnodontiformes. Pycnodontiformes also occur in younger Upper Cretaceous localities of Mexico. The occurrence of specialized teleosts (two genera of armored acanthomorphs; GonzálezRodríguez et al., 2013b) in Muhi quarry is interpreted as endemism (González-Rodríguez et al., 2013a).

Actinistian remains are new at Muhi quarry. The actinistian gulars described here were discovered in December 2013 and May 2014, respectively. Actinistians possess a pair of gulars that are interpreted as homologous to lateral gulars (Jollie, 1962, fig. 4-33; Forey, 1998, p. 270); a median gular is unknown in actinistians. Lateral gulars of actinistians are very characteristic (Hagdorn and Mutter, 2011; Hauser and Martill, 2013, fig. 6: gular plates of different osteichthyans and distinct actinistian gular) and easily identified with a little experience; for example, a teuthoid gladius in Fuchs et al. (2008) was recently reinterpreted as a gular of an actinistian by Schultze et al. (2010).

\section{Material and methods}

Material: the actinistian record of Muhi quarry is based on one left gular plate (UAHMP 3966) and on a partial gular plate associated with a basibranchial tooth plate (UAHMP 3970). The left gular plate was deposited with the outer surface on the sediment so that the deepened inner surface of the gular was filled with additional layers of sediment, which pitch out laterally as seen on the lateral side of the 


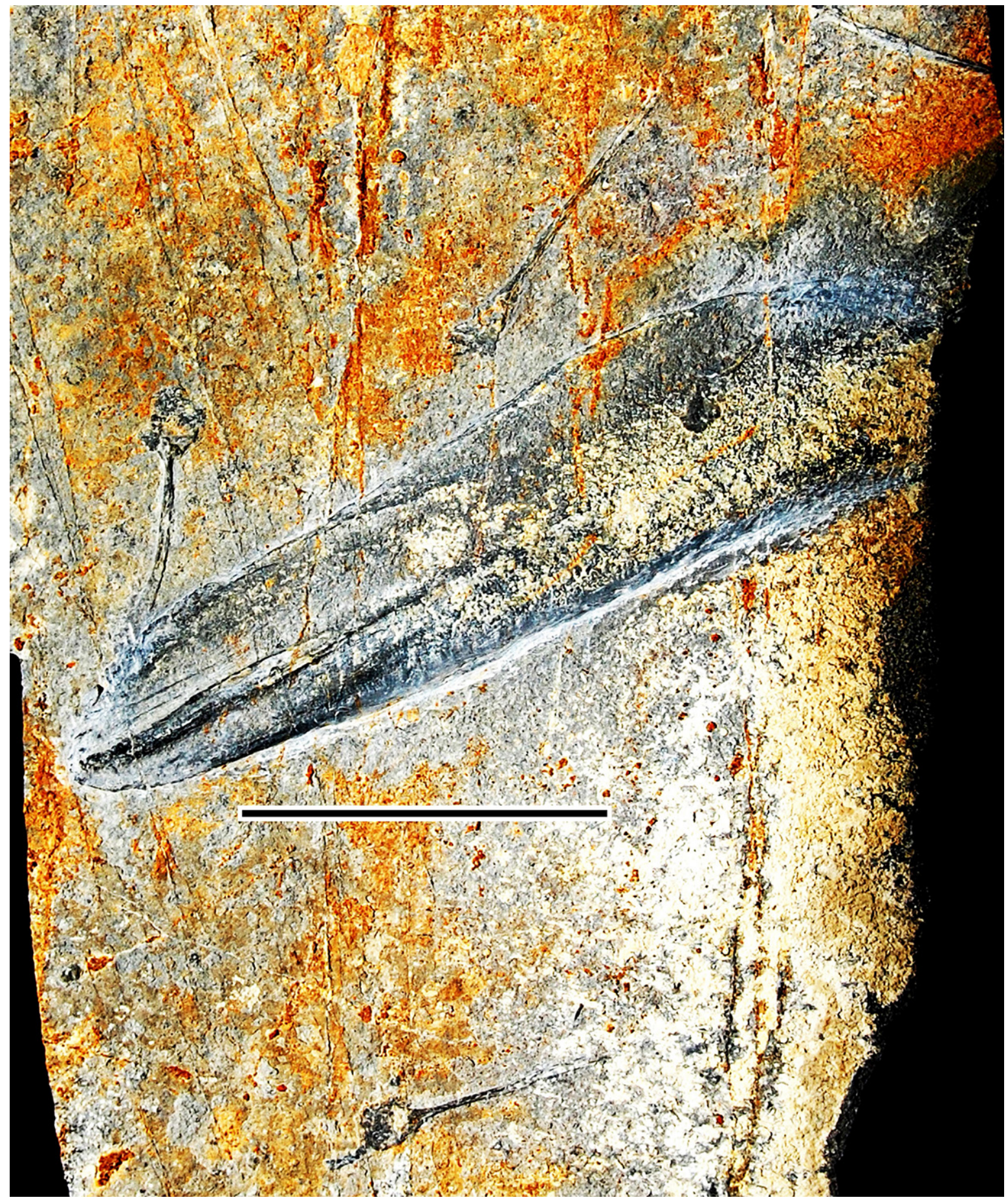

Figure 1. Limestone block UAHMP 3966 with a left lateral gular plate and five neural spines. Scale bar $10 \mathrm{~cm}$.

block. The partial gular plate is also preserved on the lower side of the block.

On both blocks, elongated elements with a broad base occur besides the gulars. They are interpreted here as neural spines. In addition, there is an isolated neural arch with a short neural spine on specimen UAHMP 4408.
Methods: measurements were taken with a caliper. Measurements of published figures were calculated from the given scale. In cases where this was not possible, authors were asked for help (see acknowledgements). Preparation of the specimens was not necessary; only the margins of the left gular plate were cleaned with a needle. Photographs were taken 

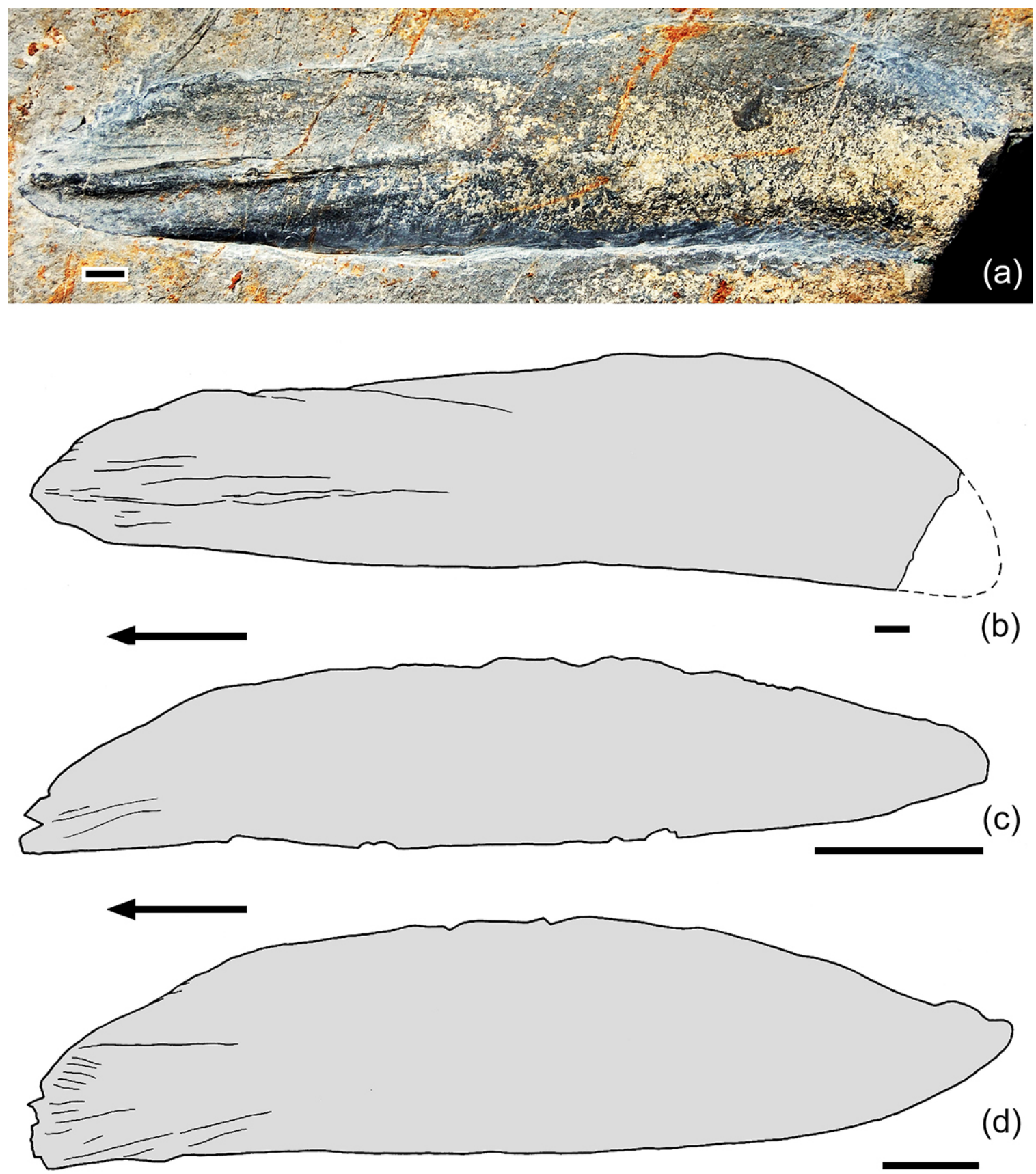

Figure 2. Left gular plate UAJMP 3966; (a) photo (scale in cm) and (b) drawing. Drawings of (c) left gular plate of Libys polypterus after Hauser and Martill (2013, fig. 5B) and of (d) left gular plate of Triassic actinistian indet. after Hauser and Martill (2013, fig. 3b). Arrows point anteriad. Scale bars $1 \mathrm{~cm}$.

with a Nikon Coolpix P4 digital camera, and drawings were made using a Wild M5A stereomicroscope with a camera lucida attachment.

Institutional abbreviations: AMNH, American Museum of Natural History, Vertebrate Collections, New York, New York, USA; KUI, University of Kansas, Natural History Museum and Biodiversity Institute, Ichthyology collection, Lawrence, Kansas, USA; KUVP, University of Kansas, Nat- ural History Museum and Biodiversity Institute, Vertebrate Paleontology collection, Lawrence, Kansas, USA; MHNM, Musée d'Histoire naturelle de Miguasha, Parc de Miguasha, Quebec, Canada; PU, former Princeton University Geological Museum, now at Peabody Museum, Yale University, New Haven, Connecticut, USA; UAHMP, Museo de Paleontología, Universidad Autónoma del Estado de Hidalgo, 
Table 1. Measurements of neural spines of the indet. actinistians from Muhi quarry.

\begin{tabular}{lllll}
\hline & $\begin{array}{l}\text { Spine } \\
\text { length }\end{array}$ & $\begin{array}{l}\text { Arch } \\
\text { length }\end{array}$ & $\begin{array}{l}\text { Center } \\
\text { diameter }\end{array}$ & Position \\
\hline Spine A & $63 \mathrm{~mm}$ & $11 \mathrm{~mm}$ & $14 \mathrm{~mm}$ & on UAHMP 3966 below gular \\
\hline Spine B & $55 \mathrm{~mm}$ & $10 \mathrm{~mm}$ & $12 \mathrm{~mm}$ & $\begin{array}{l}\text { on UAHMP 3966 above } \\
\text { and posterior to gular }\end{array}$ \\
\hline Spine C & $60 \mathrm{~mm}$ & $10 \mathrm{~mm}$ & - & $\begin{array}{l}\text { on UAHMP 3966 above } \\
\text { middle gular }\end{array}$ \\
\hline Spine D & $50 \mathrm{~mm}$ & $8-12 \mathrm{~mm}$ & $18 \mathrm{~mm}$ & $\begin{array}{l}\text { on UAHMP 3966 above } \\
\text { anterior tip of gular }\end{array}$ \\
\hline Spine E & $(44 \mathrm{~mm})$ & $9 \mathrm{~mm}$ & - & on UAHMP 3970 \\
\hline Spine F & $12 \mathrm{~mm}$ & $6 \mathrm{~mm}$ & $16 \mathrm{~mm}$ & on UAHMP 4408 \\
\hline
\end{tabular}

Pachuca, Hidalgo, Mexico; USNM, United States National Museum, Smithsonian Institution, Washington, D.C., USA.

\section{Morphological description}

\subsection{Gular plates}

On block UAHMP 3966 (Fig. 1), the left gular plate is preserved together with five elongated elements (largest $63 \mathrm{~mm}$ long). The preserved part of the gular plate (Fig. 2a-b) is $253 \mathrm{~mm}$ long; the gular plate with a reconstructed posterior end may have reached a length of $280 \mathrm{~mm}$. It is narrow, 40 or $50 \mathrm{~mm}$ wide in the anterior part (14-17\% of total length) and $62 \mathrm{~mm}$ at its greatest width ( $22 \%$ of total length). The anterior half of the gular is preserved in bone; the lateral margin continues up to three-quarters of the total length of the gular. The medial margin cannot be followed so far back because of incomplete preservation. The posterior half is eroded so that only the outline of the gular is visible.

The left gular plate (Fig. 2a-b) is seen from its ventral (outer) side. It is narrow compared to gular plates of other actinistians, pointed at its anterior end. It widens somewhat in its posterior part on the lateral side. The medial border is straight. The anterior part is flat with a strong medial ridge, which rises in height from the tip of the gular plate posteriad and continues to the wide elevation at the middle of the plate. There are two short low ridges lateral to the anterior end of the medial ridge. The flat area lateral to the medial ridge is bordered laterally by another ridge, which starts $90 \mathrm{~mm}$ posterior to the anterior tip (width of the gular plate at this point is $49 \mathrm{~mm}$ ). The lateral ridge and the flat area extend to the elevated area of the middle region. The elevated area flattens out laterad, where the gular plate is widest $(62 \mathrm{~mm})$. The gular lacks ornamentation and any trace of a pit line.

A smaller gular plate on limestone block UAHMP 3970 (Fig. 3) is only partly preserved. A section $(75 \mathrm{~mm})$ of the anterior portion is preserved. The middle ridge is prominent; it flattens posteriad. Striae run on the middle ridge and parallel to the ridge, and a few small and short ridges are close to the posterior lateral margin of the gular plate.

\subsection{Basibranchial tooth plate}

A smaller bone lies below the gular (Fig. 3); its shape is more or less triangular. Small plates with small teeth lie on the surface of the bone; we interpret this bone as a basibranchial tooth plate, by comparison with similar elements in other actinistians (e.g., Latimeria: "copula" of Smith, 1940, fig. 8; Diplurus: Schaeffer, 1952, fig. 8 and pl. 12, fig. 2; Latimeria: Nelson, 1969, fig. 14C: posterior part of the paired tooth plate series of [branchial] arch 2, pl. 81, fig. 1; Megalocoelacanthus: "posterior tooth plate of basibranchial" in Dutel et al., 2012, fig. 16A, of which the posterior end of the posterior basibranchial tooth plate is visible; Smith, 1940, pl. 23; Dutel et al., 2012, fig. 16A).

\subsection{Neural spines and arches}

Four elongated elements lie dispersed around and one on top of gular UAHMP 3966 (Fig. 1). The elements (Fig. 4a-f) have long narrow spines - up to $63 \mathrm{~mm}$ long. The spines are round in diameter above the arch but show a median furrow in more distal parts; this condition indicates that only the outside of the spine was ossified, and the center was hollow (see reconstructed cross section of neural spines of $\mathrm{Lau}^{-}$ gia groenlandica in Stensiö, 1932, fig. 20 showing a similar pattern), so that it collapsed during compaction. A small canal appears in the lower round part of the neural spine above the joint of the two parts of the neural arch; it is the canal for the dorsal ligament (Andrews, 1977, figs. 1-3). The neural arches dorsally surround an ossified rounded sheet of about 14 to $18 \mathrm{~mm}$ in depth. The notochordal sheet is either smooth or divided into small pieces. In one spine (Fig. 4ab), a hemal process (13 $\mathrm{mm}$ long) is attached to the lower side of the rounded sheet. This neural spine is interpreted 


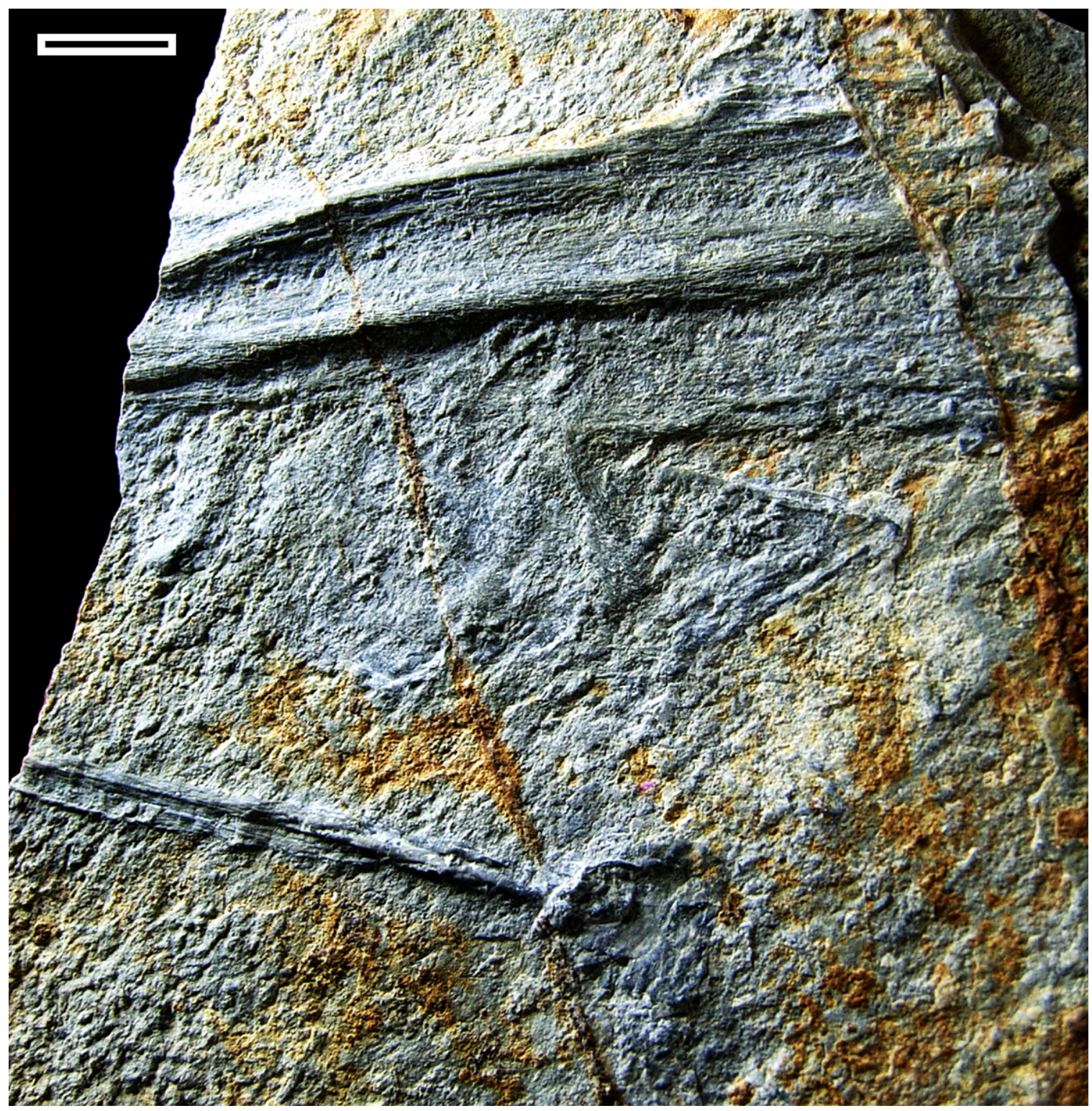

Figure 3. Specimen UAHMP 3970: partly preserved gular at the top, branchial plate with teeth below the gular, and one neural spine at the bottom. Scale bar $1 \mathrm{~cm}$.

here as belonging to the posterior abdominal region because of the short hemal process (compare with Andrews, 1977, fig. 3A). The other spines (Fig. 4c-e) cannot be placed in any specific body region. They could be spines of the abdominal or caudal region, where the notochord is narrow and the space for the neural cord minimal (Millot and Anthony, 1958, pl. 75; Andrews, 1977, fig. 4; Arratia et al., 2001, fig. 29C). There is one isolated rounded structure with a short neural spine (UAHMP 4408, Fig. 4g). By comparison with Latimeria (Millot and Anthony, 1958, pl. 50), this neural arch is interpreted as belonging to the most anterior part of the axial skeleton, close to the head. A mineralized, rounded sheet appears in place of the notochord; it is missing in the neural spine (Fig. 4c), lying above the posterior part of gular plate UAHMP 3966, preserved laterally and showing only the neural arch of both sides superimposed on each other next to the spine.

The spine (Fig. 4d) above the middle part of gular UAHMP 3966 shows the notochordal sheet divided into two half-moons. The size of these elongated elements (Table 1), interpreted as neural spines, corresponds to the size of the neural spine of Megalocoelacanthus (Dutel et al., 2012, fig. 19A), an actinistian even larger than the one of Muhi quarry. 


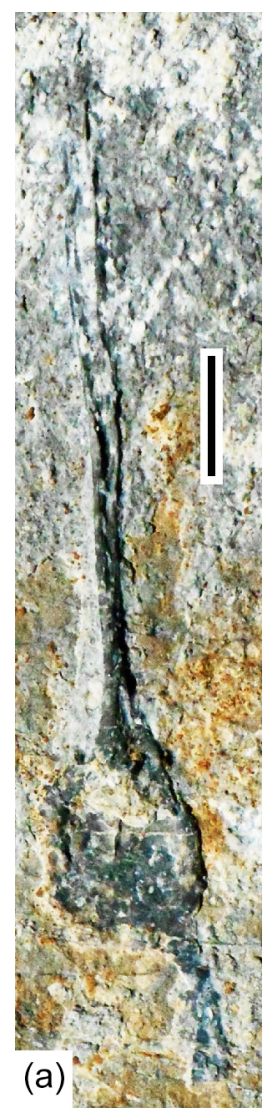

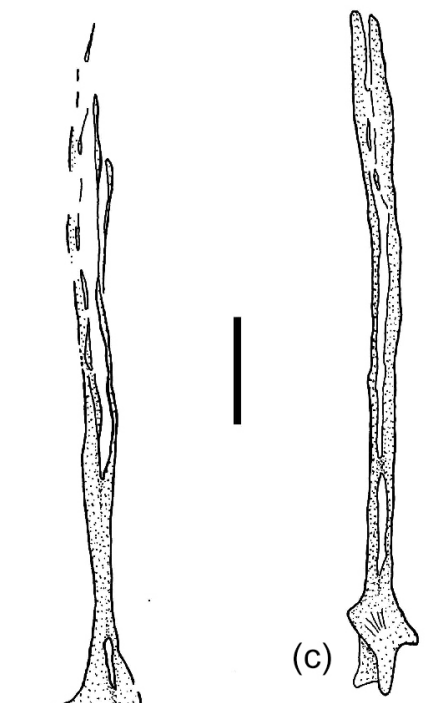

(b)

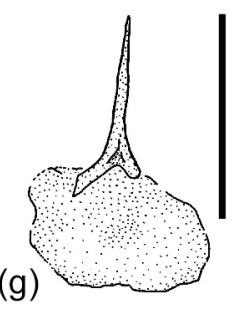

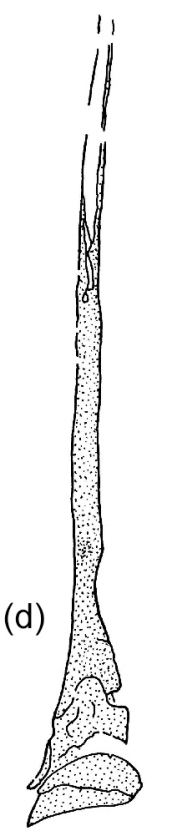
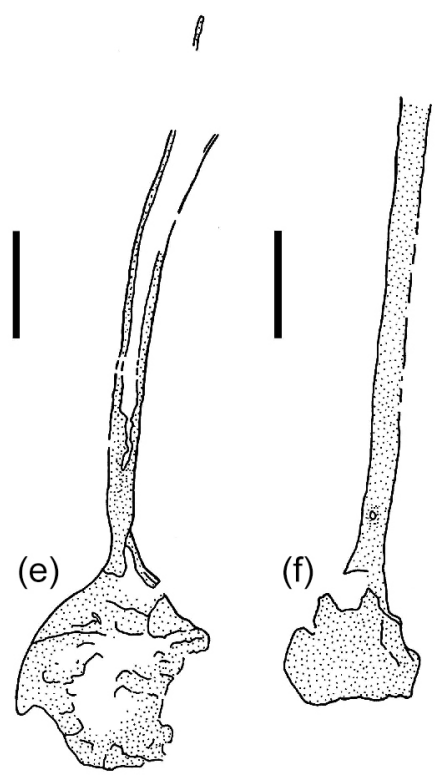

Figure 4. (a-e) Specimen UAHMP 3966: (a) neural spine with hemal process below gular plate, (b) drawing of (a, c), neural spine above posterior part of gular, (d) neural spine above middle part of gular, (e) neural spine above anterior part of gular, (f) neural spine on specimen UAHMP 3970, and (g) neural spine from the region behind the skull, UAHMP 4408. Scale bars $1 \mathrm{~cm}$.

\section{Comparison}

Gular plates have been recorded and described in many actinistians (Table 2), but they have never received special attention. They are paired and easily recognizable in fossil and living actinistians (Schultze et al., 2010; Hauser and Martill, 2013). Actinistians possess only lateral gulars; a median is missing. Thus the anterior tips of the lateral gular plates lie close together or touch each other in contrast to other sarcopterygians (except onychodonts: Jessen, 1966, fig. 6C, and Andrews et al., 2006, figs. 13, 46a, c) and primitive actinopterygians where the anterior tips of the lateral gulars form a triangular space to accept the median gular (e.g., Schultze and Campbell, 1986, fig. 5). Lateral gular plates of actinistians and onychodonts meet in a straight median line, the actinistian gulars nearly along the whole midline, whereas the onychodont gular plates have a large posterolaterally extending part. The lateral margin of the actinistian gular plates is continuously convex, often symmetrically (see Figs. 5 and 6). The lateral part overlaps the lower jaw, whereas it borders the submandibulars in other sarcopterygians. The combination of straight median margin with a continuous convex lateral margin is the tool to recognize isolated gular plates as those of actinistians.

Schaeffer $(1941,1952)$ compared different skeletal elements of many actinistian taxa, and he demonstrated differences useful for characterization of taxa. However, he did not use gulars, supposedly because they show few distinctions among taxa. Cloutier (1996, fig. 9) illustrated gulars of three Devonian actinistian genera. Schultze et al. (2010) cited records for five species and genera including the extant actinistian Latimeria chalumnae. Hauser and Martill (2013, fig. 4) figured eight gulars of seven fossil genera and one indet. Searching the literature, we found gulars of 61 species figured in external or internal view (see Table 2). In addition, 13 are illustrated in lateral view so that only the length could be measured or estimated.

Identification of left and right gulars: it is not easy to distinguish between right and left gulars if they are not found in contact. Hauser and Martill (2013, p. 983, fig. 3) described a Triassic gular as a right gular. However, they figured it as a left gular in a comparative figure (Hauser and Martill, 2013, fig. 4h). In Latimeria, the anterior pointed ends of the gulars are close to each other in the midline, whereas the poste- 
Table 2. Measurements of gular plate length and total body length of different coelacanth species. Explanations: (number) - measurement of gular length in lateral view; g - gular; 1 - length; le - left; ri - right; Sp - total length of specimen; w - width; 80 (bold) - measurement by the author; [number] - measurements taken from Hauser and Martill (2013, table 2). Remarks: Forey (1998): all figures are drawings (reconstructions) except Sassenia; Lund and Lund (1985): all figures are drawings.

\begin{tabular}{|c|c|c|c|c|c|}
\hline $\begin{array}{l}\text { Species Fig. (in this paper) } \\
\text { original reference }\end{array}$ & $\begin{array}{l}\mathrm{g}-\text { length } \\
\text { in } \mathrm{mm}\end{array}$ & $\begin{array}{l}\mathrm{g}-\text { width } \\
\text { in } \mathrm{mm}\end{array}$ & $\begin{array}{l}\text { Sp - length } \\
\text { in } \mathrm{mm}\end{array}$ & $\mathrm{g}-1 / \mathrm{g}-\mathrm{w}$ & $\mathrm{g}-1 / \mathrm{Sp}-1$ \\
\hline \multicolumn{6}{|l|}{ Alcoveria brevis Fig. $6 \mathrm{Y}$} \\
\hline Beltan (1972), pl. 1, figs. A, B & 20.3 & 4.93 & 9.7 & 4.12 & 0.255 \\
\hline \multicolumn{6}{|l|}{ Allenypterus montanus Fig. $6 \mathrm{~b}$} \\
\hline Lund and Lund (1985), figs. 60, 57 & 16.5 & 3.68 & 63.5 & 4.48 & 0.26 \\
\hline Forey (1998), fig. 4.6 & 12.25 & 2.94 & & 4.17 & \\
\hline \multicolumn{6}{|l|}{ Axelrodichthys araripensis Fig. 5M } \\
\hline Hauser and Martill (2013), tbl. 2 & [50] no scale & & {$[405]$} & & {$[0.124]$} \\
\hline Maisey (1991), fig. on p. 308 & 54.37 & & & & \\
\hline Forey (1998), fig. 4.17 & $(54.9)$ & & & & \\
\hline Forey (1998), fig. 11.3 & $(150)$ & & & & \\
\hline Maisey 3.6.15 & 94 & 21 & 710 & 4.48 & 0.324 \\
\hline \multicolumn{6}{|l|}{ Caridosuctor populosum Fig. 6B } \\
\hline Lund and Lund (1985), fig. 21 & 65.4 & 19.2 & & 3.4 & \\
\hline Lund and Lund (1985), fig. 22 & 28.8 (same specimen) & 8.65 & & 3.3 & \\
\hline Lund and Lund (1985), fig. 19 & $(19.5)$ & & 113.8 & & 0.171 \\
\hline \multicolumn{6}{|l|}{ Chagrinia enodis Fig. $6 \mathrm{~T}$} \\
\hline Cloutier (1996), fig. 9D & 24 & 7.2 & & 3.3 & \\
\hline \multicolumn{6}{|l|}{ Chinlea sorenseni Fig. $5 \mathrm{~J}$} \\
\hline \multicolumn{6}{|l|}{ Elliott (1987), fig. 2B } \\
\hline Elliott 27.1.15 & 101.8 & 27.9 & & 3.67 & \\
\hline \multicolumn{6}{|l|}{ Coccod. nudum $=$ suevicum } \\
\hline Reis (1888), pl. 3, fig. 1 & 31 & 9 & & 3.44 & \\
\hline \multicolumn{6}{|l|}{ Coccoderma substriolatum } \\
\hline Huxley (1866), pl. 10, fig. 4 & $>86$ & 29 & & $>3.1$ & \\
\hline \multicolumn{6}{|l|}{$\begin{array}{l}\text { Coccoderma } \\
\text { suevicum Fig. } 6 \mathrm{D}\end{array}$} \\
\hline Forey (1998), fig. 4.11, & 31.2 & 7.5 & & 4.16 & \\
\hline Forey (1998), fig. 5.7 & 39.8 & 10.3 & & 3.86 & \\
\hline Lambers (1992), p. 11 & & & 305 & & \\
\hline Vetter (1881), pl. 2, fig. 4 & 50 & 12 & & 4.2 & \\
\hline Coelacanthidae gen. sp. indet. Fig. $6 \mathrm{~L}$ & $22-115$ & & & & \\
\hline Hagdorn and Mutter (2011), p. 233, & 22-115 & & & & \\
\hline fig. $7 \mathrm{j}$ & 39 & 13 & & 3.0 & \\
\hline \multicolumn{6}{|l|}{ Coelacanthus granulatus Fig. 6F } \\
\hline Schaumberg (1978), fig. 4 & $48.55 / 38.7$ & $11.14 / 9.12$ & & $4.36 / 4.24$ & \\
\hline Forey (1998), fig. 11.4 & $(50.6)$ & & 404.6 & & 0.125 \\
\hline $\begin{array}{l}\text { Diplocercides heiligenstockensis Fig. 6d } \\
\text { Cloutier (1996), fig. 9C }\end{array}$ & 13.6 & 4.1 & \multicolumn{2}{|c|}{ Diplocercides heiligenstockensis Fig. 6d } & \\
\hline \multicolumn{6}{|l|}{ Diplocercides jaekeli Fig. 6S } \\
\hline Stensiö (1937), pl. 10, fig. 3 & 24.8 & $? 7.2$ & & 3.45 & \\
\hline Stensiö (1937), pl. 8, fig. 1 & 24.9 & 6.5 & & 3.83 & \\
\hline \multicolumn{6}{|l|}{ Diplocercides kayseri Fig. 60} \\
\hline Cloutier (1996), fig. 9B & 29.7 & 6.8 & & 4.37 & \\
\hline Stensiö (1937), fig. 18 & $(26)$ & & & & \\
\hline \multicolumn{6}{|l|}{ Diplocercides sp. Fig. 6I } \\
\hline Szrek (2007), fig. 4 & le $44 /$ ri 40.2 & le $10.74 /$ ri 9.22 & & $4.1 / 4.36$ & \\
\hline
\end{tabular}


Table 2. Continued.

\begin{tabular}{|c|c|c|c|c|c|}
\hline $\begin{array}{l}\text { Species Fig. (in this paper) } \\
\text { original reference }\end{array}$ & $\begin{array}{l}\mathrm{g}-\text { length } \\
\text { in } \mathrm{mm}\end{array}$ & $\begin{array}{l}\mathrm{g}-\text { width } \\
\text { in } \mathrm{mm}\end{array}$ & $\begin{array}{l}\text { Sp - length } \\
\text { in } \mathrm{mm}\end{array}$ & $\mathrm{g}-1 / \mathrm{g}-\mathrm{w}$ & $\mathrm{g}-1 / \mathrm{Sp}-1$ \\
\hline \multicolumn{6}{|l|}{ Diplurus gwyneddensis } \\
\hline Bock (1959), tbl. p. 43 & 38 & 10 & & 3.8 & \\
\hline \multicolumn{6}{|l|}{ Diplurus longicaudatus Fig. 5G } \\
\hline Bock (1959), tbl. p. 43 & 140 & 40 & & 3.5 & \\
\hline Schaeffer (1952), tbl. 1 & $(140)$ & & 640 & & 0.219 \\
\hline USNM18476 pl. 16, fig. 2 & le $143 /$ ri 140 & le $42 /$ ri 38 & & $3.4 / 3.68$ & \\
\hline \multicolumn{6}{|l|}{ Diplurus newarki Fig. 6Z } \\
\hline Schaeffer (1952), fig. 8 & 12.9 & 2.9 & & 4.45 & \\
\hline reconstruction fig. 9 & 16 & - & 140 & & 0.114 \\
\hline PU14945, tbl. 1 & & & 61.5 & & \\
\hline AMNH15222, tbl. 1 & [13] & & 86.2[159] & & 0.083 \\
\hline PU14918, tbl. 1 & [15] & & $110[80]$ & & 0.082 \\
\hline PU14943, pl. 8 & 17.5 & 3.2 & 80 & 5.47 & 0.219 \\
\hline PU14944, pl. 5, fig. 3, & (12) & & 115 & & 0.104 \\
\hline pl. 9 , fig. 1 & 12 & 2.6 & & 4.62 & \\
\hline PU14959, tbl. 1 & & & 131 & & \\
\hline Schaeffer (1941), fig. 2C, & 10.4 & 2.57 & & 4.05 & \\
\hline p. 2 & 11 & 2.7 & 110 & 4.07 & 0.100 \\
\hline Bock (1959), tbl. p. 43 & 16.5 & 3 & 140 & 5.5 & 0.118 \\
\hline Forey (1998), fig. 11.6 & $(10.8)$ & & 98.4 & & 0.110 \\
\hline \multicolumn{6}{|l|}{ Dobrogeria aegyssensis Fig. 5N } \\
\hline Cavin and Grădinaru (2014), fig. 12 & 88.6 & 29.9 & & 2.96 & \\
\hline \multicolumn{6}{|l|}{ Garnbergia ommata } \\
\hline Martin and Wenz (1984), fig. 1 & $(65)$ & & & & \\
\hline \multicolumn{6}{|l|}{ Graphiurichthys callopterus } \\
\hline Kner (1866), pl. 1, fig. 1 & 21.45 & 4.45 & 82 & 4.82 & 0.262 \\
\hline \multicolumn{6}{|l|}{ Guizhoucoelacanthus guanlingensis Fig. 5Q } \\
\hline Geng et al. (2009), fig. 1 & $(76.2)$ & & 354.8 & & 0.215 \\
\hline Geng et al. (2009), fig. 2 & 73.7 & $? 15.2$ & & $? 4.85$ & \\
\hline \multicolumn{6}{|l|}{ Hadronector donbairdi Fig. $6 \mathrm{a}$} \\
\hline Lund and Lund (1985), figs. 35/36 & 16.75 & 3.25 & 69.5 & 5.15 & 0.241 \\
\hline \multicolumn{6}{|l|}{ Holophagus gulo Fig. $6 \mathrm{~N}$} \\
\hline Forey (1998), fig. 11.8 Holophagus sp. & $(100)$ & & 589.2 & & 0.170 \\
\hline Gardiner (1960), pl. 43 , fig. 2 & 30.5 & 9 & & 3.4 & \\
\hline \multicolumn{6}{|l|}{ Indocoelacanthus robustus Fig. $5 \mathrm{H}$} \\
\hline Jain (1974), fig. 2 & $120.3 / 126.9$ & $31.7 / 34.6$ & & $3.79 / 3.67$ & \\
\hline pl. 1, fig. 1 & 131 & 37 & & 3.54 & \\
\hline Latimeria chalumnae KUI 22082 male & 148 & 48 & 972 & 3.08 & 0.152 \\
\hline \multicolumn{6}{|l|}{ Latimeria chalumnae female Fig. 5D } \\
\hline Smith (1940), p. 9, pl. 9 (?female, estimated from size) & 225 & 66 & 1434.8 & 3.4 & 0.195 \\
\hline Millot and Anthony (1958), fig. 6 & 177 & 58 & & 3.05 & \\
\hline Forey (1998), fig. 8.1 & $(281.4)$ & & 1434.8 & & 0.195 \\
\hline \multicolumn{6}{|l|}{ Latimeria chalumnae embryo } \\
\hline Forey (1998), fig. 2.3 & 86.6 & ri $25.6 / 1 \mathrm{le} 26.8$ & & $3.38 / 3.23$ & \\
\hline \multicolumn{6}{|l|}{ Laugia groenlandica Fig. 6V } \\
\hline Stensiö (1932), pl. 1, fig. 2 & 21.2 & 6 & 131.3 & 3.53 & 0161 \\
\hline Forey (1998), fig. 4.10 & $(21.8)$ & & & & \\
\hline Forey (1998), fig. 11.10 & $(25.6)$ & & 139.02 & & 0.184 \\
\hline \multicolumn{6}{|l|}{ Libys polypterus Figs. 2C. 5R } \\
\hline Reis (1888), pl. 2, fig. 2 & 71.5 & 22 & & 3.25 & \\
\hline Hauser and Martill (2013), fig. 5B LipHM & 56.9 & 11.25 & & 5.05 & \\
\hline
\end{tabular}


Table 2. Continued.

\begin{tabular}{|c|c|c|c|c|c|}
\hline $\begin{array}{l}\text { Species Fig. (in this paper) } \\
\text { original reference }\end{array}$ & $\begin{array}{l}\mathrm{g}-\text { length } \\
\text { in } \mathrm{mm}\end{array}$ & $\begin{array}{l}\mathrm{g} \text { - width } \\
\text { in } \mathrm{mm}\end{array}$ & $\begin{array}{l}\text { Sp - length } \\
\text { in } \mathrm{mm}\end{array}$ & $\mathrm{g}-\mathrm{l} / \mathrm{g}-\mathrm{W}$ & $\mathrm{g}-1 / \mathrm{Sp}-1$ \\
\hline $\begin{array}{l}\text { Lochmocercus aciculodontus Fig. } 6 \mathrm{e} \\
\text { Lund and Lund (1985), fig. } 68\end{array}$ & 12.6 & (2.4) & 73.4 & (5.3) & 0.172 \\
\hline $\begin{array}{l}\text { Luopingcoelacanthus eurylacrimalis Fig. } 6 \mathrm{~K} \\
\text { Wen et al. (2013) fig. 1A/5A } \\
\text { Wen } 2.2 .14\end{array}$ & $\begin{array}{l}53.4 / 38.3 \\
\mathbf{4 0}\end{array}$ & $\begin{array}{l}14.7 / 10.4 \\
\mathbf{1 0}\end{array}$ & $/ 150.4$ & $\begin{array}{l}3.63 / 3.68 \\
4\end{array}$ & $/ 0.255$ \\
\hline $\begin{array}{l}\text { Lualabaea lerichei } \\
\text { de Saint-Seine (1955), pl. } 2\end{array}$ & 17 & 4.5 & & 3.78 & \\
\hline $\begin{array}{l}\text { Macropoma lewesiensis } \\
\text { Forey (1998), fig. } 4.19 \\
\text { Forey (1998), fig. } 11.11\end{array}$ & $\begin{array}{l}(86.96) \\
(112.1)\end{array}$ & & 457.7 & & 0.245 \\
\hline $\begin{array}{l}\text { Macropoma mantelli Fig. } 50 \\
\text { Woodward (1909), pl. 37, fig. } 9 \\
\text { Woodward (1909), pl. 37, fig. } 10 \\
\text { Woodward (1909), fig. } 49\end{array}$ & $\begin{array}{l}68 \\
77 \\
(81)\end{array}$ & $\begin{array}{l}21 \\
19\end{array}$ & 429 & $\begin{array}{l}3.24 \\
4.05\end{array}$ & 0.189 \\
\hline $\begin{array}{l}\text { Macropoma precursor } \\
\text { Woodward (1909), pl. 38, fig. } 8\end{array}$ & (39) & & & & \\
\hline $\begin{array}{l}\text { Macropoma speciosum } \\
\text { Tima (1986), pl. IV }\end{array}$ & $(67.3 / 77.3)$ & & $375 / 431.3$ & & 0.179 \\
\hline $\begin{array}{l}\text { Macropoma willemoesii } \\
\text { Vetter (1881), pl. 1, fig. } 1\end{array}$ & (41) & & 245 & & 0.167 \\
\hline $\begin{array}{l}\text { Macropomoides orientalis } \\
\text { Forey (1998), fig. } 11.21\end{array}$ & (13) & & 63.6 & & 0.204 \\
\hline $\begin{array}{l}\text { Mawsonia brasiliensis Fig. 5F } \\
\text { Hauser and Martin (2013), tbl. } 2 \\
\text { Yabumoto (2002), fig. } 4\end{array}$ & $\begin{array}{l}{[189]} \\
176.9\end{array}$ & 50.7 & $\begin{array}{l}{[929]} \\
1006.7\end{array}$ & 3.49 & $\begin{array}{l}{[0.204]} \\
0.176\end{array}$ \\
\hline $\begin{array}{l}\text { Mawsonia gigas Fig. 5B } \\
\text { Woodward (1907) pl. 8, fig. } 5\end{array}$ & 337.5 & 91.2 & & 3.7 & \\
\hline $\begin{array}{l}\text { Mawsonia sp. Fig. } 6 \mathrm{E} \\
\text { Gallo et al. (2010), fig. } 5\end{array}$ & 49.5 & 18.4 & & 2.69 & \\
\hline $\begin{array}{l}\text { Megalocoelacanthus dobiei Fig. 5A } \\
\text { Dutel et al. (2012), fig. 14C } \\
\text { Cast } \\
\text { Maisey 3.6.15 }\end{array}$ & $\begin{array}{l}400 \\
\mathbf{4 0 4 / 4 0 3} \\
\mathbf{4 7 5}\end{array}$ & $\begin{array}{l}125.3 / 128.9 \\
\mathbf{1 3 0 / 1 2 9 . 9} \\
\mathbf{1 5 0}\end{array}$ & & $\begin{array}{l}3.19 / 3.1 \\
3.1 / 3.11 \\
3.17\end{array}$ & \\
\hline $\begin{array}{l}\text { Miguashaia bureaui Fig. 6G } \\
\text { Cloutier (1996), MHNM 06-41, fig. 9A }\end{array}$ & 48.2 & 13.8 & 195 & 3.49 & 0.247 \\
\hline $\begin{array}{l}\text { Mylacanthus lobatus } \\
\text { Stensiö (1921), pl. 18, fig. } 4\end{array}$ & $? 45$ & 19 & & $? 2.37$ & \\
\hline $\begin{array}{l}\text { Mylacanthus spinosus } \\
\text { Stensiö (1921), pl. 19, fig. } 3\end{array}$ & $>62$ & 31 & & $>2$ & \\
\hline $\begin{array}{l}\text { Palaeoctopus pelagicus Fig. 6A } \\
\text { Fuchs et al. (2008), p. 1133, fig. } 6 \\
\text { Schultze et al. (2010), p. } 690\end{array}$ & $\begin{array}{l}70 \\
66\end{array}$ & 20 & about 600 & 3.5 & 0.110 \\
\hline $\begin{array}{l}\text { Parnaibaia maranhaoensis Fig. } 6 \mathrm{H} \\
\text { Yabumoto (2008), fig. } 2 \\
\text { Yabumoto (2008), fig. } 3 \\
\text { Yabumoto (Feb. 2015) }\end{array}$ & $\begin{array}{l}47.8[56] \\
41.4[50] \\
\mathbf{4 5 . 0}[\mathbf{5 2 . 7}]\end{array}$ & $\begin{array}{l}11.9 \\
10.3[11.3] \\
\mathbf{1 0 . 8}\end{array}$ & 363.4 & $\begin{array}{l}4.0[4.7] \\
4.0[4.4] \\
4.17[4.88]\end{array}$ & 0.132 \\
\hline $\begin{array}{l}\text { Piveteauia madagascariensis Fig. 6W } \\
\text { Hauser and Martin (2013), tbl. } 2 \\
\text { Clément (1999), figs. 1, } 2\end{array}$ & $\begin{array}{l}{[23]} \\
20.4\end{array}$ & 5.9 & $\begin{array}{l}{[87]} \\
130-140\end{array}$ & 3.46 & $\begin{array}{l}{[0.269]} \\
0.151\end{array}$ \\
\hline
\end{tabular}


Table 2. Continued.

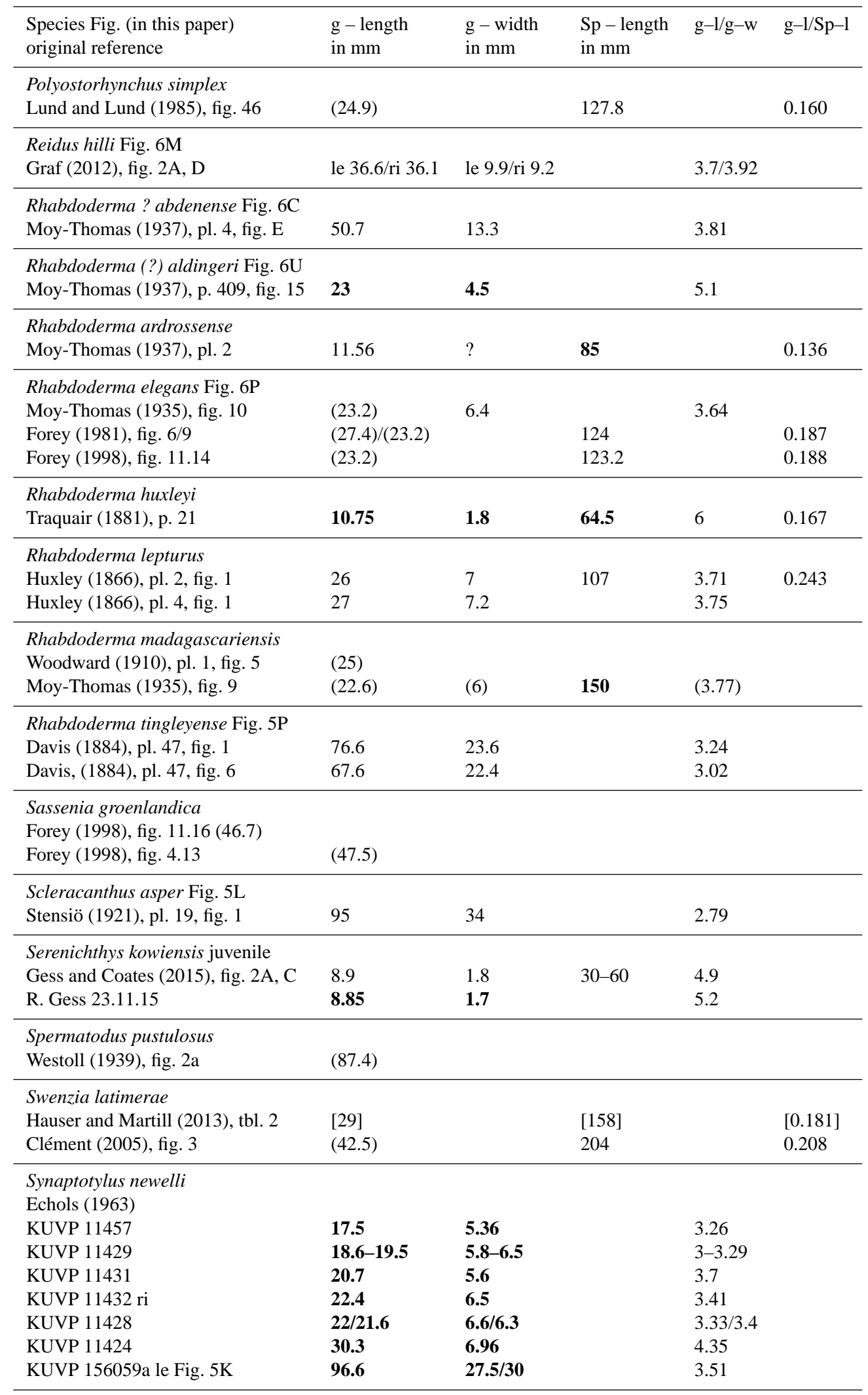


Table 2. Continued.

\begin{tabular}{|c|c|c|c|c|c|}
\hline $\begin{array}{l}\text { Species Fig. (in this paper) } \\
\text { original reference }\end{array}$ & $\begin{array}{l}\mathrm{g}-\text { length } \\
\text { in } \mathrm{mm}\end{array}$ & $\begin{array}{l}\mathrm{g}-\text { width } \\
\text { in } \mathrm{mm}\end{array}$ & $\begin{array}{l}\text { Sp - length } \\
\text { in } \mathrm{mm}\end{array}$ & $\mathrm{g}-1 / \mathrm{g}-\mathrm{w}$ & $\mathrm{g}-1 / \mathrm{Sp}-1$ \\
\hline \multicolumn{6}{|l|}{ Ticinepomis peyeri } \\
\hline Hauser and Martill (2013), tbl. 2 & [17] & & {$[125]$} & & [0.136] \\
\hline Rieppel (1980), fig. 6 & $(6.4)$ & & 44.6 & & 0.143 \\
\hline \multicolumn{6}{|l|}{ Trachymetopon liassicum Fig. 5E } \\
\hline Hennig (1951), tbl. p.70 & 210 & 55 & $\begin{array}{l}1640 \\
(1750)\end{array}$ & 3.82 & $\begin{array}{l}0.128 \\
(0.12)\end{array}$ \\
\hline Hennig (1951), pl. 8, fig. 5 & 180 & 71 & & 2.54 & \\
\hline Hauff and Hauff (1981), fig. 70 & 170 & 66 & 1317.5 & 2.58 & 0.181 \\
\hline Dutel et al. (2015), p. 2 & & & 1600 & & \\
\hline Dutel et al. (2015), fig. 1A & $? 238$ & & $>1700$ & & \\
\hline Dutel et al. (2015), fig. 3B & 168.5 & 69.7 & & 2.42 & \\
\hline \multicolumn{6}{|l|}{ Triassic coelacanth indet. Figs. 2D, 5I } \\
\hline Hauser and Martill (2013), figs. 3a, b, 4h & 110.2 & 24.9 & & 4.42 & \\
\hline \multicolumn{6}{|l|}{ Undina acutidens Fig. 6J } \\
\hline Reis (1892), pl. 2, fig. 10 & 43 & 10.8 & 255 & 3.98 & 0.169 \\
\hline \multicolumn{6}{|l|}{ Whiteia nielseni Fig. 6X } \\
\hline Forey (1998), fig. 5.9D & 20.4 & 5.5 & & 3.71 & \\
\hline \multicolumn{6}{|l|}{ Whiteia tuberculata Fig. 6C } \\
\hline Forey (1998), fig. 5.9F & 10.1 & 2.8 & & 3.6 & \\
\hline Lehman (1952), fig. 14 & (17) & & & & \\
\hline Lehman (1952), pl. 4, fig. C & 14.5 & 4.4 & & 3.3 & \\
\hline \multicolumn{6}{|l|}{ Whiteia woodwardi Fig. 6R } \\
\hline Lehman (1952), fig. 13 & (24) & & 127 & & 0.189 \\
\hline Lehman (1952), fig. 9A & 26.4 & 7.6 & & 3.47 & \\
\hline Lehman (1952), pl. 1 & 25.6 & 7.3 & & 3.5 & \\
\hline Lehman (1952), pl. 2, fig. C & 25 & 8.6 & & 2.9 & \\
\hline Forey (1998), fig. 4.15 & $(32.8)$ & & & & \\
\hline Forey (1998), fig. 11.18 & $(32.4)$ & & 146.7 & & 0.221 \\
\hline \multicolumn{6}{|l|}{ Youngichthys xinghuainsis } \\
\hline Wang and Liu (1981), fig. 3 & $(10)$ & & 132 & & \\
\hline \multicolumn{6}{|l|}{ Muhi quarry spec. } \\
\hline UAHMP 3966 Figs. 2A, B, 5C & 253-280 & 62 & & 4.52 & \\
\hline
\end{tabular}

rior ends diverge from each other (Smith, 1940, pl. 9). By comparison with Latimeria, we prefer to orient the gulars of Megalocoelacanthus (Fig. 5) in the opposite way to Dutel et al. (2012, fig. 14C), reflecting the condition in Latimeria. The same morphology can be seen in Libys polypterus (Hauser and Martill, 2013, fig. 5A, B), Macropoma mantelli (Woodward, 1909, pl. 37, fig. 9), Undina acutidens (Reis, 1892, pl. 2, fig. 10), and Whiteia woodwardi (Lehman, 1952, fig. 9A), where both gulars are preserved in situ. In Reidus, both gulars are close to each other along the whole midline and separated a little only at their most posterior part (Graf, 2012, fig. 2A, D).

Gular size: the length of the gulars is correlated with the body length (Fig. 7). Most fossil actinistians and their gulars are small (Figs. 5 and 6); only a few fossil actinistians and their gulars (Fig. 5e-f) are comparable in size with $L a$ - timeria chalumnae and its gulars (Fig. 5d). The gular from Muhi quarry (Fig. 5c) corresponds closely to the length of the gular of Latimeria chalumnae (Fig. 7), whereas the gulars of Mawsonia gigas and of Megalocoelacanthus dobiei (Fig. 5ab) are longer than the gular of Latimeria chalumnae. Naturally, size changes with growth (compare gular length given by Forey, 1998, for an embryo of Latimeria of $86.6 \mathrm{~mm}$ with that of $225 \mathrm{~mm}$ in adults; Table 2). The same can be seen in fossil actinistians (e.g., Synaptotylus from 17.5 to $96.6 \mathrm{~mm}$ in length; Table 2). Hagdorn and Mutter (2011) cited gulars of 22 to $115 \mathrm{~mm}$ length from one Triassic geological horizon and interpreted them as growth stages of one species. Figure 6 shows that most gulars are small, because most fossil actinistians are small. The gular of Serenichthys kowiensis (Gess and Coates, 2015) is the smallest gular of all figured gulars; the specimens are interpreted as juvenile by the au- 


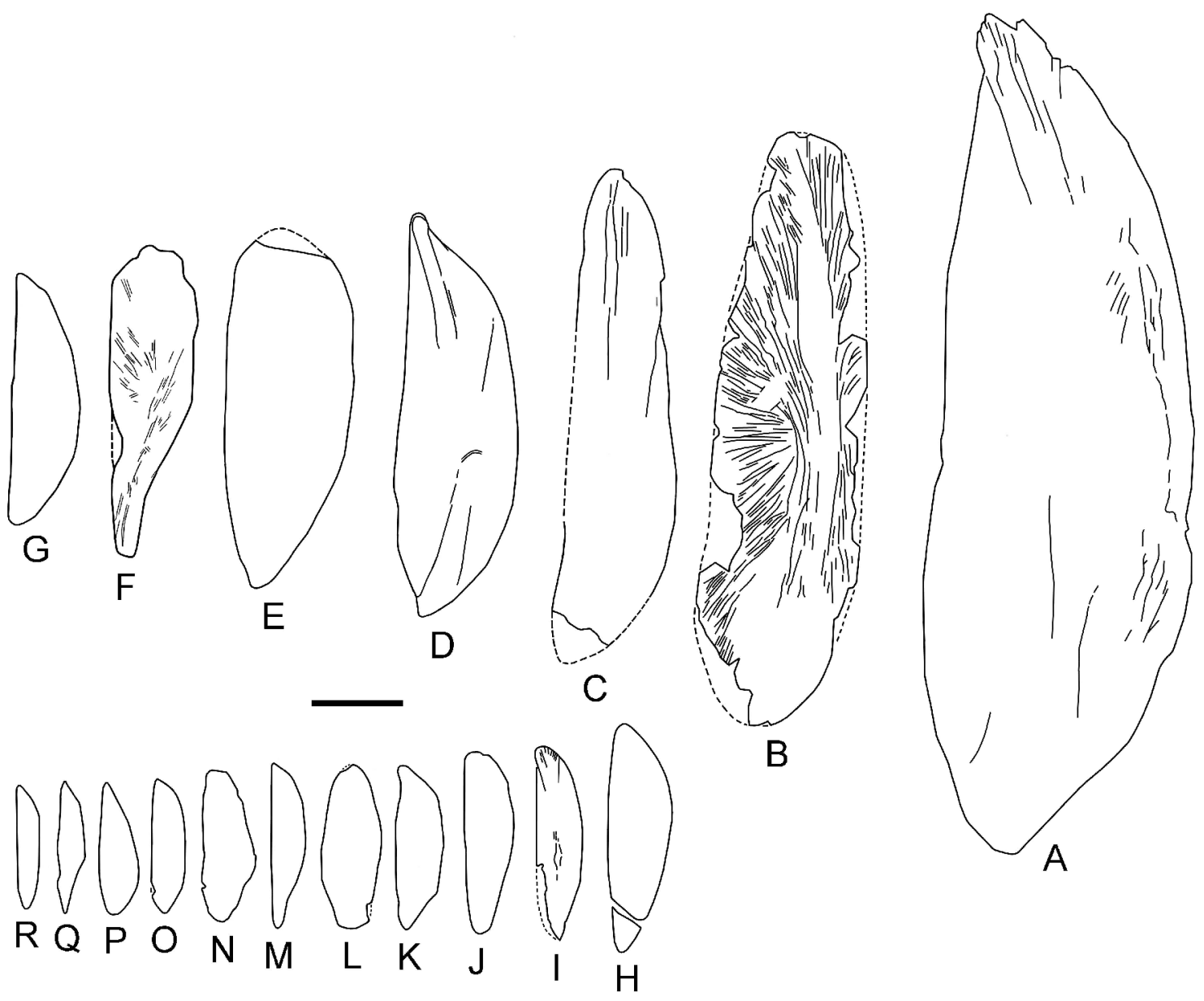

Figure 5. Large left gular plates of actinistians in external view in descending size: (A) Megalocoelacanthus dobiei (Dutel et al. 2012, fig. 14C; size after measurement of J. Maisey), (B) Mawsonia gigas (Woodward, 1907, pl. 8, fig. 5), (C) Muhi gular UAHMP 3966, (D) Latimeria chalumnae (female, Smith, 1940, p. 9, pl. 9), (E) Trachymetopon liassicum (Hennig, 1951, pl. 8, fig. 5), (F) Mawsonia brasiliensis (Yabumoto, 2002, fig. 4). (G) Diplurus longicaudatus (Bock, 1959, fig. 6B), (H) Indocoelacanthus robustus (Jain, 1974, fig. 2), (I) Triassic coelacanth (Hauser and Martill, 2013, fig. 3a, b), (J) Chinlea sorensensis (Elliott, 1987, fig. 2B), (K) Synaptotylus newelli (large specimen KUVP 156059a), (L) Scleracanthus asper (Stensiö, 1921, pl. 19, fig. 1), (M) Axelrodichthys araripensis (Maisey, 1991, fig. on p. 308), (N) Dobrogeria aegyssensis (Cavin and Grădinaru, 2014, fig. 12), (O) Macropoma mantelli (Woodward, 1909, pl. 37, fig. 10), (P) Rhabdoderma tingleyensis (Davis, 1884, pl. 47, fig. 1), (Q) Guizhoucoelacanthus guanlingensis (Geng et al., 2009, fig. 2), (R) Libys polypterus (Reis, 1888, pl. 2, fig. 2). Scale bar $5 \mathrm{~cm}$.

thors. In Table 2 and Fig. 7, the largest gular was used in each case to compare with or estimate the length of the fish.

In addition, sexual differences should be considered as shown by Latimeria chalumnae, since adult females (max: $2 \mathrm{~m}$ long) are larger than males (max: $1.68 \mathrm{~m}$ long) in the extant coelacanth Latimeria. This proportional length ratio between Latimeria female and male is also proportionally present in the length of their gular plates (Table 2: length of female Latimeria gular $225 \mathrm{~mm}$ versus length of male Latimeria gular $148 \mathrm{~mm}$ ). Unfortunately, the distinction between sexes is not possible in most fossils, because there are no external features distinguishing the sexes. The gular of the Muhi actinistian belongs to the exceptionally long gulars. The left Muhi gular is longer than those of Latimeria chalumnae listed in Table 2. Only the gulars of Mawsonia gigas and Megalocoelacanthus dobiei are longer than that of the Muhi actinistian. In the relationship of gular length to body length, the Muhi gular corresponds to a body length of about 1.62-1.64 m (Fig. 7). All large actinistians with a body length above $1 \mathrm{~m}$ are from the Cretaceous and Recent and one (Trachymetopon) from the Jurassic; they belong to either the Latimeriidae or Mawsoniidae. The Triassic Wimania (family Whiteiidae) may reach a size over $1 \mathrm{~m}$ also, but only the upper part of the head is known (Stensiö, 1921), whereas gular and body are unknown.

The length / width ratio (g-1/g-w) of gulars varies from 2.5 (Trachymetopon liassicum) to 6 (Rhabdoderma huxleyi) in actinistians. Most g-l/g-w values lie between 3 and 4 (Ta- 


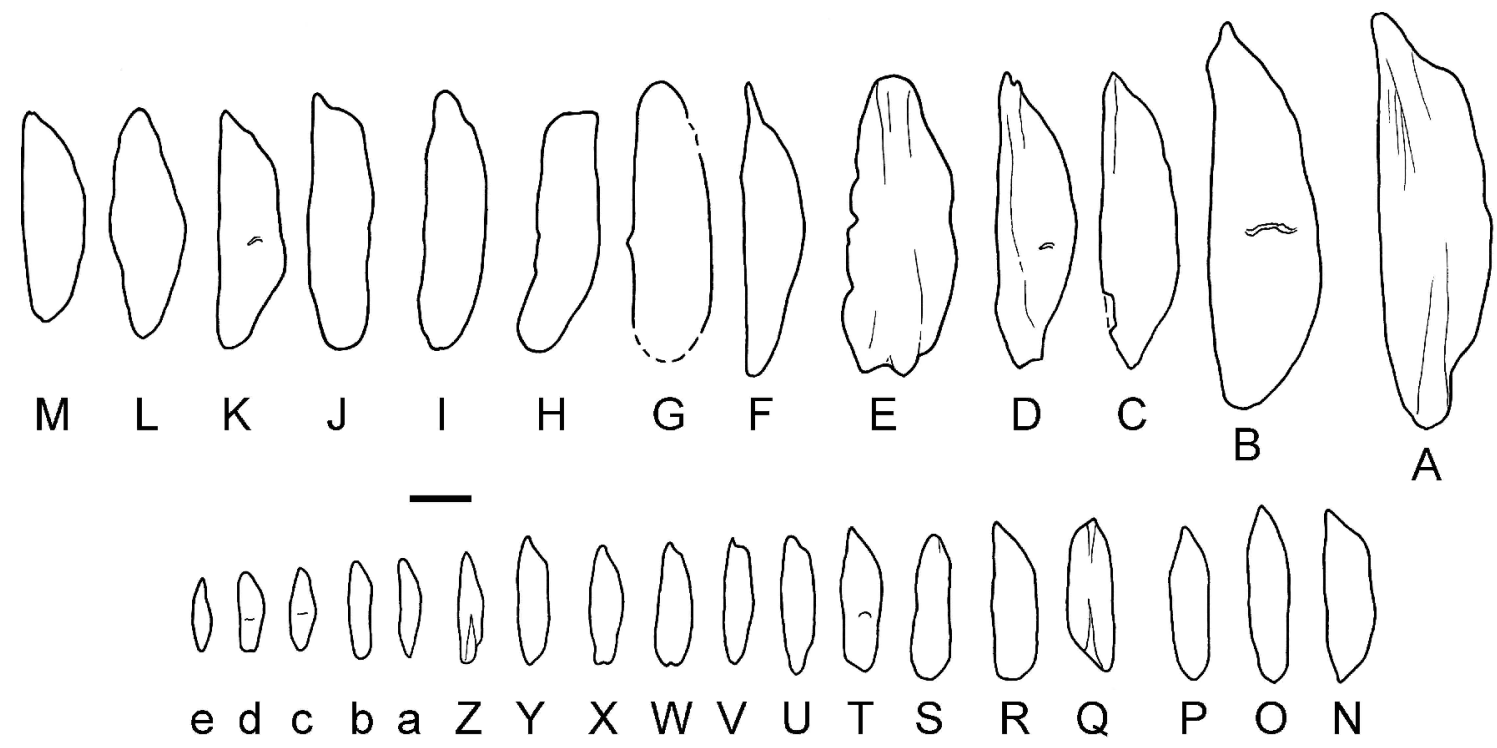

Figure 6. Small left gular plates in external view in descending size: (A) Palaeoctopus pelagicus (Fuchs et al., 2008, fig. 6A), (B) Caridosuctor populosum (Lund and Lund, 1985, fig. 21), (C) Rhabdoderma? abdenense (Moy-Thomas, 1937, pl. 4, fig. E), (D) Coccoderma suevicum (Forey, 1998, fig. 5.7, Vetter, 1881, pl. 2, fig. 4), (E) Mawsonia sp. (Gallo et al., 2010, fig. 5), (F) Coelacanthus granulatus (Schaumberg, 1978, fig. 4), (G) Miguashaia bureaui (Cloutier, 1996, fig. 9A), (H) Parnaibaia maranhaoensis (Yabumoto, 2008, fig. 3), (I) Diplocercides sp. (Szrek, 2007, fig. 4), (J) Undina acutidens (Reis, 1892, pl. 2, fig. 10), (K) Luopingcoelacanthus eurylacrimalis (Wen et al., 2013, fig. 1), (L) Coelacanthidae gen. indet. (Hagdorn and Mutter, 2011, fig. 7j), (M) Reidus hilli (Graf, 2012, fig. 2A), (N) Holophagus sp. (Gardiner, 1960, pl. 43, fig. 2), (O) Diplocercides kayseri (Cloutier, 1996, fig. 9B), (P) Rhabdoderma elegans (Moy-Thomas, 1935, fig. 10), (Q) Rhabdoderma lepturus (Huxley, 1866, pl. 4, fig. 2), (R) Whiteia woodwardi (Lehman, 1952, fig. 9A), (S) Diplocercides jaekeli (Stensiö, 1937, pl. 10, fig. 3), (T) Chagrinia enodis (Cloutier, 1996, fig. 9D), (U) Rhabdoderma? aldingeri (Moy-Thomas, 1937, fig. 15), (V) Laugia groenlandica (Stensiö, 1932, pl. 3, fig. 2), (W) Piveteaui madagascariensis (Clément, 1999, fig. 2), (X) Whiteia nielseni (Forey, 1998, fig. 5.9D), (Y) Alcoveria brevis (Beltan, 1972, pl. 1, fig. A), (Z) Diplurus newarki (Schaeffer, 1941, fig. 2C), (a) Hadronector donbairdi (Lund and Lund, 1985, fig. 35), (b) Allenypterus montanus (Lund and Lund, 1985, fig. 60), (c) Whiteia tuberculata (Forey, 1998, fig. 5.9F), (d) Diplocercides heiligenstockensis (Cloutier, 1996, fig. 9C), (e) Lochmocercus aciculodontus (Lund and Lund, 1985, fig. 68). Scale bar $1 \mathrm{~cm}$.

ble 2; Fig. 8). Few gulars (12 species and 2 unnamed species) are narrow with $\mathrm{g}-1 / \mathrm{g}-\mathrm{w}$ values between 4 and 5 as in the gular from Muhi quarry, and there are even fewer with g-1/g-w values over 5 (five species including the Libys polypterus specimen of Hauser and Martill, 2013, fig. 5B, which falls outside the normal size range of L. polypterus; Fig. 2C herein). The narrowness is not correlated with body length, nor with any systematic trait. All species with values over 5, except a Libys polypterus specimen figured by Hauser and Martill (2013, fig. 5B), are Paleozoic forms. The Libys polypterus specimen of Hauser and Martill (2013, fig. 5B) is different $(\mathrm{g}-1 / \mathrm{g}-\mathrm{w}=5.05)$ from specimens usually identified as Libys polypterus with values of 3.25 (Table 2). The Triassic actinistian gular of Hauser and Martill (2013: g-l/g-w=4.42; Fig. 2d), the Cretaceous Axelrodichthys araripensis $(\mathrm{g}-1 / \mathrm{g}-\mathrm{w}=4.48)$, the Carboniferous Allenypterus montanus $(\mathrm{g}-1 / \mathrm{g}-\mathrm{w}=4.48)$, and the Triassic Diplurus newarki $(\mathrm{g}-1 / \mathrm{g}-\mathrm{w}=4.45)$ show the closest values to the gular of the Muhi actinistian (g-1/g-w = 4.52). Most gulars with values between 4 and 5 belong to Paleozoic or Triassic actinistians; only two are from the Cretaceous, $A x$ elrodichthys and Parnaibaia, which are both members of the family Mawsoniidae, to which also the Triassic Diplurus newarki belongs. The gular plates of the two Cretaceous species Axelrodichthys araripensis (Fig. 5m) and Parnaibaia maranhaoensis (Fig. 6h) present a shape different from that of the Muhi actinistian (Fig. 5c). There are Triassic gulars with comparable length / width values (the mawsoniid Diplurus newarki, Fig. 6z; the Triassic gular figured by Hauser and Martill, 2013, fig. 3; Graphiurichthys callopterus, Kner, 1866, pl. 1, fig. 1; and Guizhoucoelacanthus guanlingensis, Fig. 5R, with narrower gulars and higher values). The length/width ratio of the Muhi gular compares best with that of the Triassic actinistian figured by Hauser and Martill (2013; Fig. 2d herein).

Comparison with body length: the length of the Muhi gular lies between that of Mawsonia gigas and Latimeria chalumnae; it corresponds to a body length comparable to Latimeria chalumnae and Trachymetopon liassicum (Fig. 7). Nevertheless, Mawsonia gigas, Latimeria chalumnae, and Trachymetopon liassicum do not have long and narrow gulars ( $\mathrm{g}$ $1 /$ gw values of $3.7,3.1-3.4$ and $2.5-3.8$, respectively) similar to the Muhi actinistian. Gular shape does not correlate with body length. Greater body length is found in Mesozoic 


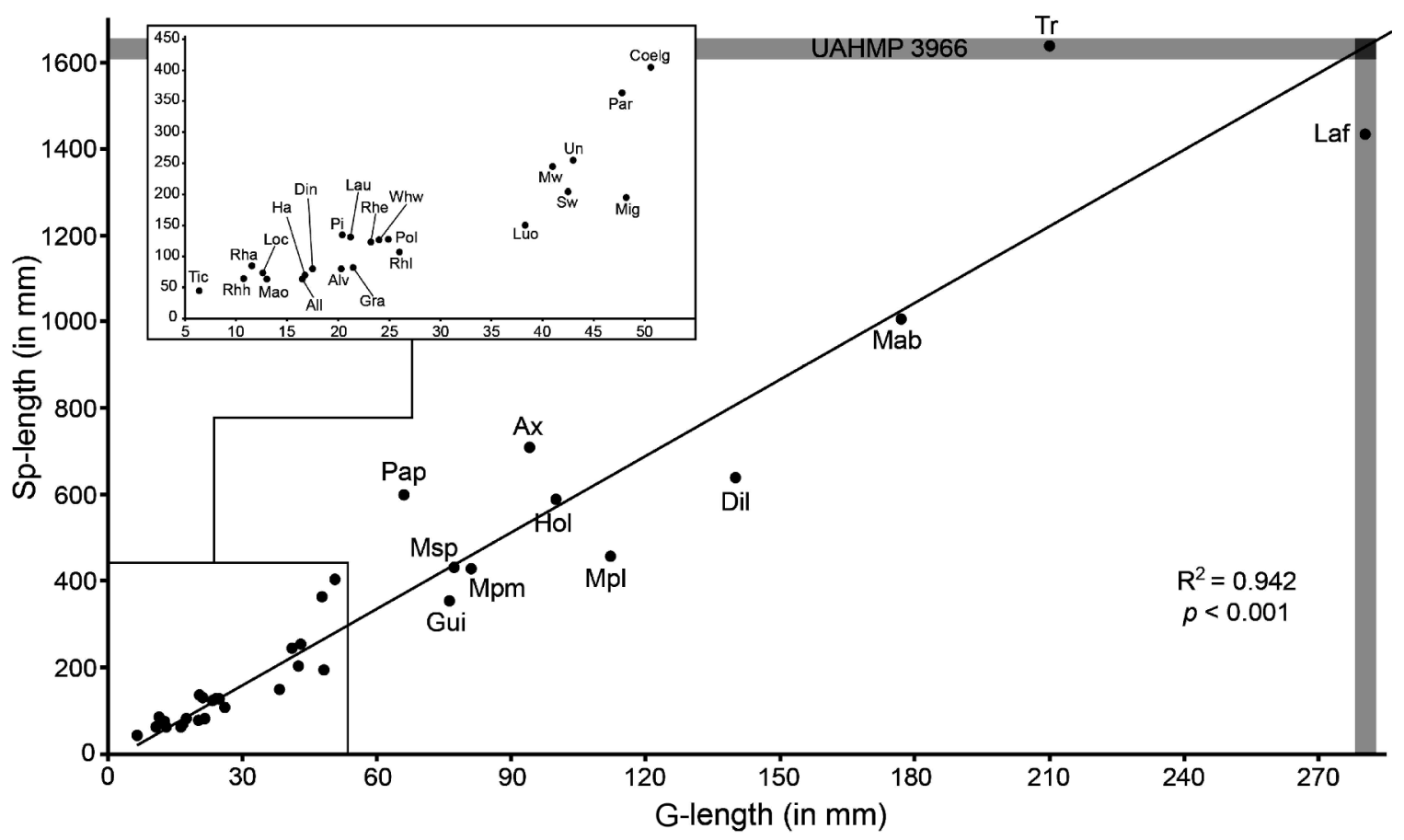

Figure 7. Correlation of gular length with body length: the length of the Muhi gular (253 to $280 \mathrm{~mm}$ ) corresponds to a body length of 1620 to 1640 mm. Abbreviations: All - Allenypterus montanus; Alv - Alcoveria brevis; Ax - Axelrodichthys araripensis; Coelg - Coelacanthus granulatus; Dil - Diplurus longicaudatus; Din - Diplurus newarki; Gra - Graphiurichthys callopterus; Gui - Guizhoucoelacanthus guanlingensis; Ha - Hadronector donbairdi; Hol - Holophagus gulo; Laf - Latimeria chalumnae female; Lau - Laugia groenlandica; Loc - Lochmocercus aciculodontus; Luo - Luopingcoelacanthus eurylacrimalis; Mab - Mawsonia brasiliensis; Mao - Macropomoides orientalis; Mig - Miguashaia bureaui; Mpl - Macropoma lewesiensis; Mpm - Macropoma mantelli; Msp - Macropoma speciosum; Mw Macropoma willemoesii; Pap - Palaeoctopus pelagicus; Par - Parnaibaia maranhaoensis; Pi - Piveteauia madagascariensis; Pol - Polyosteorhynchus simplex; Rha - Rhabdoderma ardrossense; Rhe - Rhabdoderma elegans; Rhh - Rhabdoderma huxleyi; Rhl - Rhabdoderma lepturus; Sw - Swenzia latimerae; Tic - Ticinepomis peyeri; Tr - Trachymetopon liassicum; Un - Undina acutidens; Whw - Whiteia woodwardi; UAHMP 3966 - Muhi gular.

and Recent actinistians, but it occurs in both families, Mawsoniiidae and Latimeriidae. The Muhi gular is narrow with a widening posterior portion (Fig. 2a-b). A similar gular shape occurs in two Paleozoic forms, Rhabdoderma tingleyensis (Fig. 5p) and Caridosuctor populosum (Fig. 6b); the gulars of both Paleozoic species have a length / width ratio between 3 and 4 and cannot be considered narrow gulars.

Neural spines: the elongated elements around the gular are interpreted as neural arches even though they do not compare closely with the three-forked neural spines typical of fossil actinistians (Arratia et al., 2001, figs. 31, 32). In addition, unusual for actinistians, there appears to be a mineralized, rounded sheet in the place of the notochord. The size of these elongated elements (Table 1), interpreted as neural spines, corresponds to the size of the neural spine of Megalocoelacanthus (Dutel et al., 2012, fig. 19A), an actinistian even larger than the one of Muhi quarry. The arch is bent and does not possess two straight halves as in most actinistians (e.g., Huxley, 1866, pl. 5, figs. 1, 2, 57; Stensiö, 1932, fig. 20C, E, pl. 2, fig. 2, pl. 4, pl. 6, figs. 1, 2, pl. 7, fig. 1, pl. 8; Schaeffer, 1952, pl. 5, figs. 13, pl. 6, fig. 1, pl. 9, fig. 1; Lund and Lund, 1985, figs. 2, 3, 15, 19, 35, 46, 57; Yabumoto, 2008, fig. 5, and many more). The features of the elongated elements compare with neural spines of large actinistians like Latimeria and Megalocoelacanthus.

\section{Conclusions}

The left gular plate found in the Muhi quarry belongs to a large actinistian of about $1.6 \mathrm{~m}$. It is a supplementary taxon within the supposedly Albian fish fauna of the Muhi quarry in the Mexican state of Hidalgo. The shape of the gular, its length / width ratio, and the estimated body length are not diagnostic enough to refer these Muhi gular plates to any actinistian genus. Tentatively the new form is placed within the Mawsoniidae, because similar length / width ratios of the gular occur in three genera of Mawsoniidae: Axelrodichthys, Diplurus, and Parnaibaia. This is proposed with great caution, because shape and length/width ratio of gulars and body size are not correlated with a taxonomic unit within ac- 


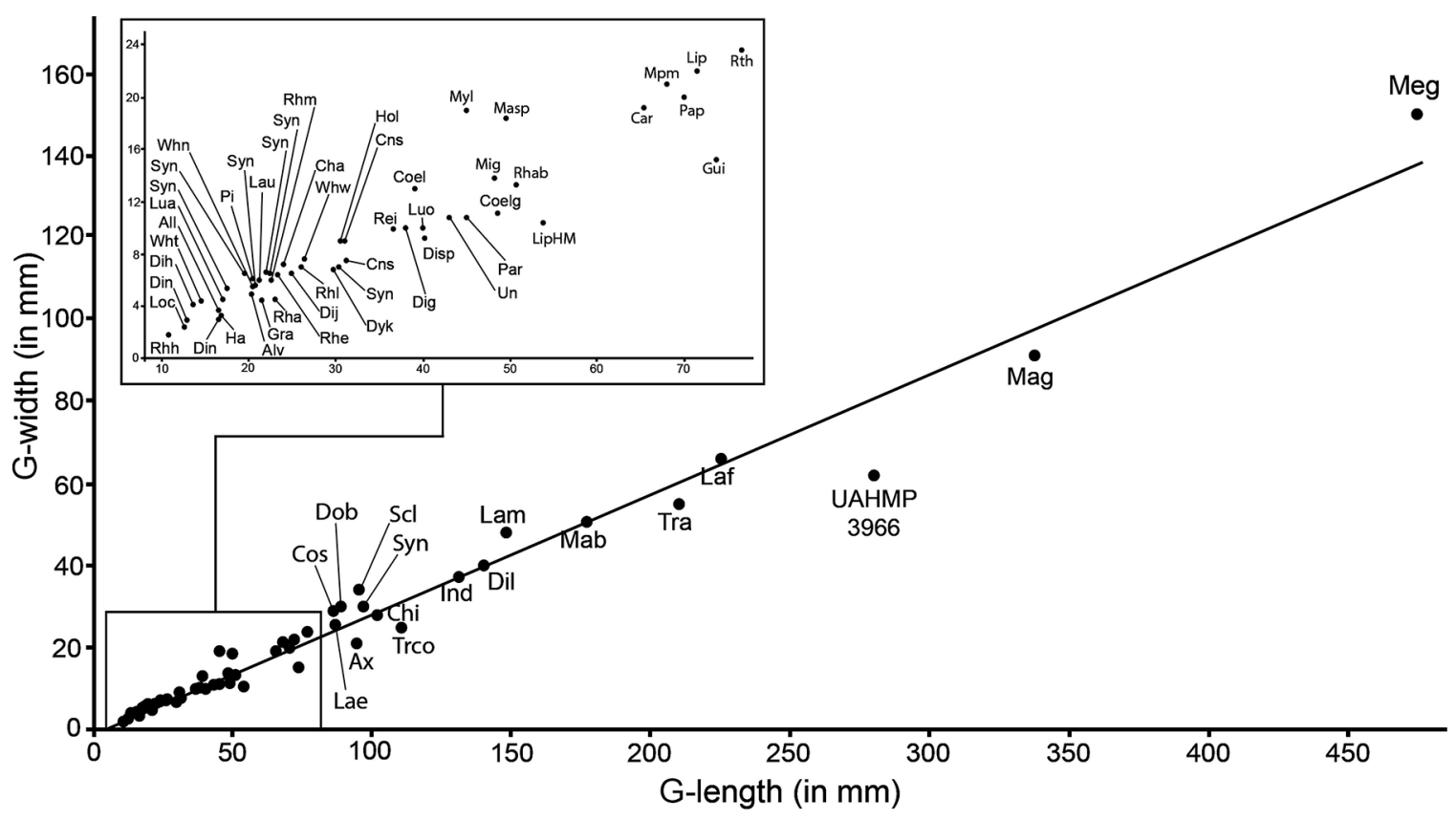

Figure 8. Correlation of gular width with gular length: UAHMP 3966 below regression line indicates a narrow gular comparable to the gulars of the Triassic coelacanth figured by Hauser and Martill (2013), Diplurus newarki, Graphiurichthys and Guizhoucoelacanthus, the Jurassic Libys figured by Hauser and Martill (2013) and the Cretaceous Axelrodichthys. Abbreviations: All - Allenypterus montanus; Alv - Alcoveria brevis; Ax - Axelrodichthys araripensis; Car - Caridosuctor populosum; Cha - Chagrinia enodis; Chi - Chinlea sorenseni; Cns - Coccoderma suevicum (two specimens); Coel - ?Coelacanthidae gen. sp. indet. (Hagdorn and Mutter, 2011); Coelg - Coelacanthus granulatus; Cos - Coccoderma substriolatum; Dig - Diplurus gwyneddensis; Dih - Diplocercides heiligenstockensi; Dij - Diplocercides jaekeli; Dil Diplurus longicaudatus; Din - Diplurus newarki; Disp - Diplocercides sp.; Dob - Dobrogeria aegyssensis; Dyk - Diplocercides kayseri; Gra - Graphiurichthys callopterus; Gui - Guizhoucoelacanthus guanlingensis; Ha - Hadronector donbairdi; Hol - Holophagus gulo; Ind Indocoelacanthus robustus; Lam - Latimeria chalumnae male; Lae - Latimeria chalumnae embryo; Laf - Latimeria chalumnae female; Lau - Laugia groenlandica; Lip - Libys polypterus; LipHM - Libys polypterus figured by Hauser and Martill (2013); Loc - Lochmocercus aciculodontus; Lua - Lualabaea lerichei; Luo - Luopingcoelacanthus eurylacrimalis; Mab - Mawsonia brasiliensis; Mag - Mawsonia gigas; Masp - Mawsonia sp.; Meg - Megalocoelacanthus dobiei; Mig - Miguashaia bureaui; Mpm - Macropoma mantelli; Myl - Mylacanthus lobatus; Pap - Palaeoctopus pelagicus; Par-Parnaibaia maranhaoensis; Pi - Piveteauia madagascariensis; Rei - Reidus hilli; Rha - Rhabdoderma (?) aldingeri; Rhab - Rhabdoderma ? abdenense; Rhe - Rhabdoderma elegans; Rhh - Rhabdoderma huxleyi; Rhl - Rhabdoderma lepturus; Rhm - Rhabdoderma madagascariensis; Rth - Rhabdoderma tingleyense; Scl - Scleracanthus asper; Sw - Swenzia latimerae; Syn - Synaptotylus newelli (different specimens); Tra - Trachymetopon liassicum; Trco - Triassic coelacanth indet. (Hauser and Martill, 2013); Un - Undina acutidens; Whn - Whiteia nielseni; Wht - Whiteia tuberculata; Whw - Whiteia woodwardi; UAHMP 3966 - Muhi gular.

tinistians. Large size is until now only known from Triassic (Wimania) and younger actinistians.

The elongated elements associated with the gulars interpreted as neural spines are different from the three-forked neural spines typical of fossil actinistians. Their features compare with neural spines of large Cretaceous and Recent actinistians like Megalocoelacanthus and Latimeria.

Acknowledgements. We thank Sergio Yáñez and Victoria Labra (Zimapán, Hidalgo, Mexico) for the help in collecting the specimens. Measurements of published actinistians were taken from figured specimens. In some cases, when this was not possible or it was ambiguous, we asked colleagues for assistance. We thank especially R. Gess (Johannesburg, South Africa), D. Elliott (Flagstaff, Arizona, USA), J. Maisey (New York, USA), W. Wen (Chengdu, China), and Y. Yabumoto (Kitakyushu, Japan) for sending us mea- surements of certain gular plates. We also contacted L. Hauser and D. Martill (both at Portsmouth, UK) concerning measurements of certain published specimens. U. Göhlich (Vienna, Austria) was so kind as to help us to get pictures of Macropoma speciosum (Reuss, 1857); the publication is available on the Internet, but the plates were photocopied folded. We thank A. Bentley (Lawrence, Kansas) for access to a male Latimeria specimen. J.-P. Mendau (Berlin, Germany) executed the final line drawings based on the original illustrations done by the senior author. T. J. Meehan (Lawrence, Kansas) prepared the illustrations for electronic submission. We thank J. Priego (Pachuca) for helping with the statistical analysis. G. Arratia, T. J. Meehan, two anonymous reviewers, and G. Clément (Paris) read and corrected the manuscript.

Edited by: F. Witzmann

Reviewed by: G. Clément and two anonymous referees 


\section{References}

Andrews, S. M.: The axial skeleton of the coelacanth, Latimeria, in: Problems in Vertebrate Evolution, edited by: Andrews, S. M., Miles, R. S., and Walker, A. D., Linn. Soc. Symp. Ser. 4, Academic Press, London, 271-288, 1977.

Andrews, S. M., Long, J., Ahlberg, P. E., Barwick, R., and Campbell, K. S. W.: The structure of the sarcopterygian Onychodus jandemarrai n. sp. from Gogo, Western Australia: with a functional interpretation of the skeleton, T. Roy. Soc. Edinb. Earth Sci., 96, 197-307, 2006.

Arratia, G., Schultze, H.-P., and Casciotta, J.: Vertebral column and associated elements in dipnoans and comparison with other fishes: Development and homology, J. Morphol., 250, 101-172, 2001.

Beltan, L.: La faune ichthyologique du Muschelkalk de la Catalogne, Mem. Real Acad. Cienc. Art. Barcelona Terc Ep., 41, 281-325, 1972.

Bock, W.: New eastern American Triassic fishes and Triassic correlations, Natl. Acad. Sci. Geol. Center Res. Ser., Philadelphia, 1, 1-184, 1959.

Cavin, L., and Grădinaru, E.: Dobrogeria aegyssensis, a new early Spathian (Early Triassic) coelacanth from North Dobrogea (Romania), Acta Geol. Polon., 64, 139-145, 2014.

Clément, G.: The actinistian (Sarcopterygii) Piveteauia madagascariensis Lehman from the Lower Triassic of northwestern Madagascar: a redescription on the basis of new material, J. Vert. Paleontol., 19, 234-242, 1999.

Clément, G.: A new coelacanth (Actinistia, Sarcopterygii) from the Jurassic of France, and the question of the closest relative fossil to Latimeria, J. Vert. Paleontol., 25, 481-491, 2005.

Cloutier, R.: The primitive actinistian Miguashaia bureaui Schultze (Sarcopterygii), in: Devonian Fishes and Plants of Miguasha, Quebec, Canada, edited by: Schultze, H.-P. and Cloutier, R., Verlag Dr. Friedrich Pfeil, München, 227-247, 1996.

Davis, J. W.: On a new species of Coelacanthus (C. tingleyensis) from the Yorkshire Cannel Coal, Trans. Linn. Soc. Lond. Ser. Zool., 2, 427-433, 1884.

de Saint-Seine, P.: Poissons fossiles de l'étage de Stanleyville (Congo belge). I. La faune des argilites et schistes bitumineux, Ann. Mus. Roy. Congo Belge Tervuren, Sér. in $8^{\circ}$, Sci. Géol., 14, 1-126, 1955.

Dutel, H., Maisey, J. G., Schwimmer, D. R., Janvier, P., Herbin, M., and Clément, G.: The giant Cretaceous coelacanth (Actinistia, Sarcopterygii) Megalocoelacanthus dobiei Schwimmer, Stewart \& Williams, 1994, and its bearing on Latimerioidei interrelationships, PLOS ONE, 7, e49911, doi:10.1371/journal.pone.0049911, 2012.

Dutel, H., Herbin, M., and Clément, G.: First occurrence of a mawsoniid coelacanth in the Early Jurassic of Europe, J. Vert. Paleontol., 35, e929581, doi:10.1080/02724634.2014.929581, 2015.

Echols, J.: A New Genus of Pennsylvanian Fish (Crossopterygii, Coelacanthiformes) from Kansas, Univ. Kansas Publ. Mus. Natur. Hist., 12, 475-501, 1963.

Elliott, D. K.: A new specimen of Chinlea sorensensi from the Chinle Formation, Dolores River, Colorado, J. Arizona-Nevada Acad. Sci., 22, 47-52, 1987.

Espinosa-Arrubarrena, L., Applegate, S. P., and GonzálezRodríguez, K. A.: The first Mexican record of a coelacanth (Osteichthyes: Sarcopterygii) from the Tlayua quarries near Tepexi de Rodríguez, Puebla, with a discussion on the importance of this fossil, in: Sixth North Amer. Paleontol. Convention, Abstr. Pap., edited by: Repetski, J. E., Paleontol. Soc. Spec. Publ., 8, 116, 1996.

Forey, P. L.: The coelacanth Rhabdoderma in the Carboniferous of the British Isles, Palaeontology, 24, 203-229, 1981.

Forey, P. L.: History of the coelacanth fishes, Chapman \& Hall, London, 419 pp., 1998.

Fuchs, D., Ifrim, C., and Stinnesbeck, W.: A new Palaeoctopus (Cephalopoda: Coleoidea) from the Late Cretaceous of Vallecillo, North-eastern Mexico, and its implications on the evolution of Octopoda, Palaeontology, 51, 1129-1139, 2008.

Gallo, V., de Cavalho, M. M. S., and Santos, R. S. S.: New occurrence of $†$ Mawsoniidae (Sarcopterygii, Actinistia) in the Morro do Chaves Formation, Lower Cretaceous of the Sergipe-Alagoas Basin, Northeastern Brazil, Bol. Mus. Paraense Emilio Goeldi Ciênc. Natur. Belém, 5, 195-205, 2010.

Gardiner, B. G.: A revision of certain actinopterygian and coelacanth fishes, chiefly from the Lower Lias, Bull. Brit. Mus. (Natur. Hist.) Geol., 4, 239-384, 1960.

Geng, B.-H., Zhu, M., and Jin, F.: A revision and phylogenetic analysis of Guizhoucoelacanthus (Sarcopterygii, Actinistia) from the Triassic of China, Vertebrate PalAsiatica, 47, 165-177, 2009.

Gess, R. W. and Coates, M. I.: Fossil juvenile coelacanths from the Devonian of South Africa shed light on the order of character acquisition in actinistians, Zool. J. Linnean. Soc., 175, 360-383, doi:10.1111/zoj.12276, 2015.

González-Rodríguez, K. A., Espinosa-Arrubarrena, L., and González-Barba, G.: An overview of the Mexican fossil fish record, in: Mesozoic Fishes 5 - Global Diversity and Evolution, edited by: Arratia, G., Schultze, H.-P., and Wilson, M. V. H., Verlag Dr. F. Pfeil, München, 9-34, 2013a.

González-Rodríguez, K. A., Schultze, H.-P., and Arratia, G.: Miniature armored acanthomorph teleosts from the Albian/Cenomanian (Cretaceous) of Mexico, in: Mesozoic Fishes 5 - Global Diversity and Evolution, edited by: Arratia, G., Schultze, H.-P., and Wilson, M. V. H., Verlag Dr. F. Pfeil, München, 457-487, 2013b.

Graf, J., A new Early Cretaceous coelacanth from Texas, Histor. Biol., 24, 441-452, 2012.

Hagdorn, H. and Mutter, R. J.: The vertebrate fauna of the Lower Keuper Albertibank (Erfurt Formation, Middle Triassic) in the vicinity of Schwäbisch Hall (Baden-Württemberg, Germany), Palaeodiversity, 4, 223-243, 2011.

Hauff, B. and Hauff, R. B.: Das Holzmaden-buch, Selbstverlag, Holzmaden/Teck, 136 pp., 1981.

Hauser, L. M. and Martill, D. M.: Evidence for coelacanths in the Late Triassic (Rhaetian) of England, Proc. Geols. Assoc., 124, 982-987, 2013.

Hennig, E.: Trachymetopon liassicum Ald., ein RiesenCrossopterygier aus Schwäbischem Ober-Lias, N. Jb. Geol. Paläontol. Abh., 94, 67-79, 1951.

Huxley, T. H.: Figures and descriptions illustrative of British organic remains. Dec. 12: Illustrations on the structures of the crossopterygian ganoids, Mem. Geol. Surv. UK, London, UK, $1-44,1866$.

Jain, S. L.: Indocoelacanthus robustus n. gen., n. sp. (Coelacanthidae, Lower Jurassic), the first fossil coelacanth from India, J. Paleontol., 48, 49-62, 1974. 
Jessen, H. L.: Die Crossopterygier des Oberen Plattenkalkes (Devon) der Bergisch-Gladbach-Paffrather Mulde (Rheinisches Schiefergebirge) unter Berücksichtigung von amerikanischem und europäischem Onychodus-Material, 1966, Ark. Zool., 18, 305-391, 1966.

Jollie, M.: Chordate Morphology, Reinhold Publishing Corporation, New York, xiv, 478 pp., 1962.

Kner, R.: Die Fische der bituminösen Schiefer von Raibl in Kärnthen, Sitzber. Akad. Wiss. Math.-Naturwiss. Cl. Wien, 53, 152$197,1866$.

Lambers, P. H.: On the ichthyofauna of the Solnhofen Lithographic Limestone (Upper Jurassic, Germany), Doctoral thesis, University of Groningen, X, p. 336, 1992.

Lehman, J.-P.: Étude complémentaire des poisons de l'Eotrias de Madagascar, Kgl. Sv. VetenskAkad. Handl., 2, 1-201, 1952.

Lund, R. and Lund, W. L.: Coelacanths from the Bear Gulch Limestone (Namurian) of Montana and the evolution of the Coelacanthiformes, Bull. Carnegie Mus. Natur. Hist., 25, 1-74, 1985.

Maisey, J. G.: Axelrodichthys Maisey, 1986, in: Santana Fossils; an illustrated atlas, edited by: Maisey, J. G., TFH Publications, Neptune, New Jersey, 303-315, 1991.

Martin, M. and Wenz, S.: Découverte d'un nouveau coelacanthé, Garnbergia ommata n. g., n. sp., dans le Muschelkalk supérieur du Baden-Württemberg, Stuttgarter Beitr. Naturkde. B, 105, 1177, 1984.

Millot, J. and Anthony, J.: Anatomie de Latimeria chalumnae. Tome I. Squelette, muscles et formations de soutien, Centre National de la Recherche Scientifique, Paris, 122 pp., 1958.

Moy-Thomas, J. A.: The coelacanth fishes from Madagascar, Geol. Mag., 72, 213-227, 1935.

Moy-Thomas, J. A.: The Carboniferous coelacanth fishes of Great Britain and Ireland, Proc. Zool. Soc. Lond. B, 107, 383-415, 1937.

Nelson, G. J.: Gill arches and the phylogeny of fishes, with notes on the classification of vertebrates, Bull. Amer. Mus. Natur. Hist., 141, 475-552, 1969.

Reis, O. M.: Die Coelacanthinen, mit besonderer Berücksichtigung der im Weissen Jura Bayerns vorkommenden Gattungen, Palaeontographica, 35, 1-94, 1888.

Reis, O. M.: Zur Osteologie der Coelacanthinen. I. Theil (Rumpfskelett, Knochen des Schädels und der Wangen, Kiemenbogenskelet, Schultergürtel, Becken, Integument und innere Organe), Inaug. Diss., Philos. Fak. Kgl. Ludwigs-MaximiliansUniv. München, 1888, 1-39, 1892.

Reuss, A. E.: Neue Fischreste aus dem böhmischen Pläner, Denkschr. Akad. Wiss. Wien, Math.-Naturwiss. Cl., 13, 33-42, 1857.

Rieppel, O.: A coelacanth fish from the Middle Triassic of Monte San Giorgio, Switzerland, Eclogae Geologicae Helvetiae, 73, 921-939, 1980.

Schaeffer, B.: A revision of Coelacanthus newarki and notes on the evolution of the girdles and basal plates of the median fins in the Coelacanthini, Amer. Mus. Novitates, 1110, 1-17, 1941.

Schaeffer, B.: The Triassic coelacanth fish Diplurus, with observations on the evolution of the Coelacanthini, Bull. Amer. Mus. Natur. Hist., 99, 29-78, 1952.
Schaumberg, G.: Neubeschreibung von Coelacanthus granulatus Agassiz (Actinistia, Pisces) aus dem Kupferschiefer von Richelsdorf (Perm, W.-Deutschland), Paläontol. Z., 52, 169-197, 1978.

Schultze, H.-P. and Campbell, K. S. W.: Characterization of the dipnoi, a monophyletic group, J. Morphol., 1, 25-37, 1987.

Schultze, H.-P., Fuchs, D., Giersch, S., Ifrim, C., and Stinnesbeck, W.: Paleoctopus pelagicus from the Turonian of Mexico reinterpreted as a coelacanth (Sarcopterygian) gular plate, Palaeontology, 53, 689-694, 2010.

Smith, J. L. B.: A living coelacanthid fish from South Africa, Trans. Roy. Soc. S. Afr., 28, 1-106, 1940.

Stensiö, E. A.: son: Triassic Fishes from Spitzbergen. Part 1, Holzhausen, A., Vienna, xxvii, 307 pp., 1921.

Stensiö, E. A:son: Triassic Fishes from East Greenland, Meddel. Grønland, 83, 305 pp., 1932.

Stensiö, E. A.: son: On the Devonian coelacanthids of Germany with special reference to the dermal skeleton, Kgl. Sv. VetenskAkad. Handl., 16, 1-56, 1937.

Szerk, P.: Coelacanths (Actinistia, Sarcopterygii) from the Famennian (Upper Devonian) of the Holy Cross Mountains, Poland, Acta Geologica Polonica, 57, 403-413, 2007.

Tima, V.: Revision of Macropoma speciosum Reuss, 1857 (Crossopterygii, Coelacanthiformes). Vestnik Ústrdniho Ustavu Geol. Praha, 61, 209-216, 1986.

Traquair, R. H.: Report on the fossil fishes collected by the Geological Survey of Scotland in Eskdale and Liddesdale. Pt. 1 Ganoidei, Trans. Roy. Soc. Edinburgh, 30, 15-71, 1881.

Vetter, B.: Die Fische aus dem lithographischen Schiefer im Dresdener Museum, Mitt. Kgl. Mineral.-Geol. Mus. Prähistor. Slg. Dresden, 4, 1-118, 1881

Wang, N., and Liu, H.: [Coelacanth fishes from the marine Permian of Zhejiang, South China], Vertebrata Palasiatica, 19, 305-312, 1981.

Wen, W., Zhang, Q.-Y., Hu, S.-X., Benton, M. J., Zhou, C.-Y., Tao, X., Huang, J.-Y., and Chen, Z.-Q.: Coelacanths from the Middle Triassic Luoping Biota, Yunnan, South China, with the earliest evidence of ovoviviparity, Acta Palaeontol. Polon., 58, 175-193, 2013.

Westoll, T. S.: On Spermatodus pustulosus Cope, a coelacanth from the "Permian" of Texas, Amer. Mus. Novitates, 1017, 1-23, 1939.

Woodward, A. S.: On the Cretaceous formation of Bahia (Brazil), and on vertebrate fossils collected therein. II. The vertebrate fossils, Q. J. Geol. Soc. Lond., 63, 131-139, 1907.

Woodward, A. S.: The Fossil Fishes of the English Chalk, Part 5, Palaeontographical Society, London, 153-184, 1909.

Woodward, A. S.: On some Permo-Carboniferous fishes from Madagascar, Ann. Mag. Natur. Hist., 5, 1-6, 1910.

Yabumoto, Y.: A new coelacanth from the Lower Cretaceous of Brazil (Sarcopterygii, Actinistia), Paleontol. Res., 6, 343-350, 2002.

Yabumoto, Y.: A new Mesozoic coelacanth from the Lower Cretaceous of Brazil (Sarcopterygii, Actinistia), Paleontol. Res., 12, 329-343, 2008. 